\title{
WestVirginiaUniversity
}

THE RESEARCH REPOSITORY @ WVU

Graduate Theses, Dissertations, and Problem Reports

2016

\section{Adherence to Sport Rehabilitation}

Kjersti A. Traaen

Follow this and additional works at: https://researchrepository.wvu.edu/etd

\section{Recommended Citation}

Traaen, Kjersti A., "Adherence to Sport Rehabilitation" (2016). Graduate Theses, Dissertations, and Problem Reports. 6824.

https://researchrepository.wvu.edu/etd/6824

This Thesis is protected by copyright and/or related rights. It has been brought to you by the The Research Repository @ WVU with permission from the rights-holder(s). You are free to use this Thesis in any way that is permitted by the copyright and related rights legislation that applies to your use. For other uses you must obtain permission from the rights-holder(s) directly, unless additional rights are indicated by a Creative Commons license in the record and/ or on the work itself. This Thesis has been accepted for inclusion in WVU Graduate Theses, Dissertations, and Problem Reports collection by an authorized administrator of The Research Repository @ WVU. For more information, please contact researchrepository@mail.wvu.edu. 
Adherence to Sport Rehabilitation

Kjersti A. Traaen, ATC/L

\begin{abstract}
Thesis submitted to the College of Physical Activity and Sports Sciences

At West Virginia University

In partial fulfillment-of the requirements

For the degree of
\end{abstract}

\author{
Masters of Science \\ In \\ Athletic Training
}

Michelle A. Sandrey, PhD, ATC, Chair Damien Clement, PhD, ATC, CC-AASP, NCC

Drue Stapleton, PhD, L-ATC, CSCS

Department of Sport Sciences

Morgantown, West Virginia

2016

Keywords: Adherence, Rehabilitation, Sport Injury

Copyright 2016 Kjersti Traaen 


\section{ABSTRACT \\ Adherence to Sport Rehabilitation}

Kjersti Traaen, ATC/L

Context: Sport injuries occur in National Collegiate Athletic Association (NCAA) sanctioned sports during preseason, in season, and post season competition. In order for optimal return to play outcomes, rehabilitation programs must be implemented and enforced. However, the literature reports inconsistent adherence rates and few studies have evaluated adherence rates by including both the athlete and athletic trainer at the Division III level. Objective: The purpose of this study was to determine if there was a difference in adherence rates to rehabilitation between a pre and posttest for a Division III institution. Design: The study was a prospective exploratory study. Setting: The testing took place at a Division III institution. Only one clinician administered the testing. Patients and Other Participants: Athletes ( $n=12)$ from three teams or multiple teams and Athletic Trainers ( $\mathrm{n}=3)$ at a Division III institution volunteered for the study. Inclusion criteria for athletes included sustaining a musculoskeletal injury resulting in withdrawal from sport participation for ten or more days in order to complete rehabilitative activities, athletes must be at least 18 years of age or older, with a physical on file, be a member of a sport team, and participating in practices and/or games prior to injury. Inclusion criteria for athletic trainers included being a practicing licensed athletic trainer at the Division III institution working with team and individual sport athletes. Exclusion criteria for athletes included not having a musculoskeletal injury, rehabilitation resulting in less than ten days missed, being younger than 18 years, not having a physical on file, and not being a member of a sport team. Exclusion criteria for athletic trainers included not being licensed in the state of Pennsylvania and not working with sport teams or individual athletes at the Division III institution.

Interventions: The athlete participants were asked to complete a demographic survey, athletic identity measurement scale (AIMS), and the Rehabilitation Over Adherence Questionnaire (ROAQ) during the initial meeting. The ROAQ was administered following a pre and post-test format. The athletic trainer participants competed the Sport Injury Rehabilitation Adherence Scale (SIRAS) and the Rehabilitation Adherence Measure for Athletic Training (RAdMAT) during the initial visit and at the end of data collection. Data collection as measured by the questionnaires was held over a three-week period. Main Outcomes Measures: The dependent variable is adherence based on responses to the questionnaire. Results: Using three separate 1x2 repeated measures ANOVA, ROAQ for the athlete was not significant $(\mathrm{F}=2.382, \mathrm{P}=0.151)$, nor were the SIRAS $(\mathrm{F}=.786, \mathrm{P}=0.394)$, and RAdMAT $(\mathrm{F}=.592, \mathrm{P}=0.458)$ for the athletic trainer. There was a moderate correlation for the AIMS and ROAQ pre-test $(\mathrm{r}=3.14, \mathrm{P}=.320)$ and posttest $(\mathrm{r}=.319, \mathrm{P}=.313)$ along with a moderate correlation for the years of participation and the AIMS ( $\mathrm{r}=.353, \mathrm{P}=.261)$. A large, significant correlation for the SIRAS total and the RAdMAT total ( $r=.901, \mathrm{P}>0.01)$ was evident. Conclusions: Based on the information gathered through this prospective exploratory study, athletes and athletic trainers report similar scores regarding adherence to sport injury rehabilitation over the course of a three-week period. Athletic trainers are in a special position in being able to work with athletes daily in the athletic training room. In order to have optimal return to play outcomes, athletes must be adherent to the sport injury rehabilitation protocol, which is prescribed by the athletic trainer. 


\section{ACKNOWLEDGEMENTS}

\section{To my family}

Thomas Traaen, Donna Traaen, and Ashton Traaen

Thank you for your love and support throughout my academic career. I would not have been able to conquer this academic endeavor without your words of encouragement and motivation. Here's to an average guy having an above average day.

To Dr. Sandrey

A special thank you to my committee chair, Dr. Michelle Sandrey. Thank you for the countless meeting, drafts, and support throughout this process. I would not have been able to produce a quality piece of literature without your guidance.

To Dr. Clement

Thank you to committee member, Dr. Damien Clement. Thank you for volunteering your valuable time to serve as a committee member. Your expertise was invaluable in the creation of this paper.

To Dr. Stapleton

A special thank you to committee member, Dr. Drue Stapleton. Thank you for being excited about research, and being a readable resource. You were instrumental in providing insightful detail into this manuscript.

To my roommates

Jamie Tasco, Taylor Wade, and Neil Ehlers

Thank you for putting up with me for the past two years, and talking me off the ledge when I was certain that I wasn't going to graduate. I cannot wait to see what the next step has in store for all of you.

To my classmates

Thank you for the phenomenal two years at West Virginia University. Everyone has such a bright future, and I cannot wait to see what everyone does in the next phase of life. I look forward to reunions to hear all about the wonderful impacts and life changes that happen after graduation.

\section{To Fleetwood Mac}

Thank you for giving me my sanity back after countless hours of editing. 


\section{TABLE OF CONTENTS}

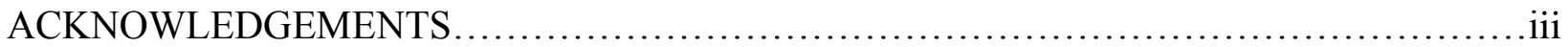

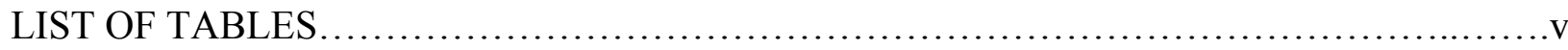

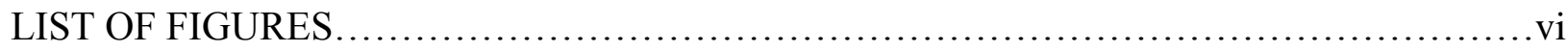

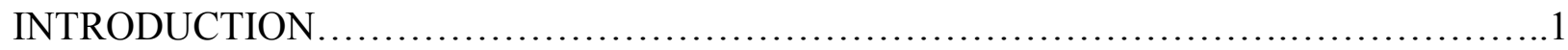

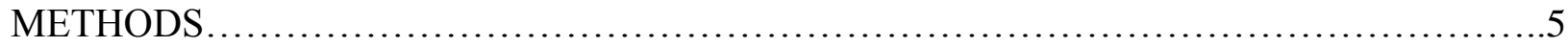

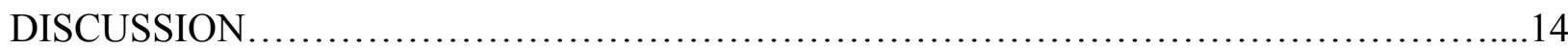

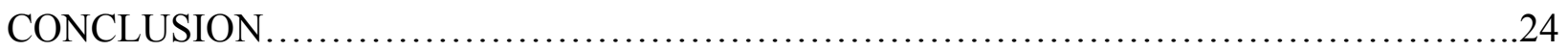

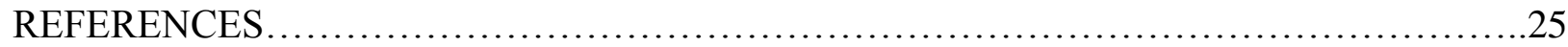

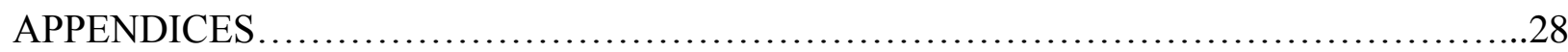

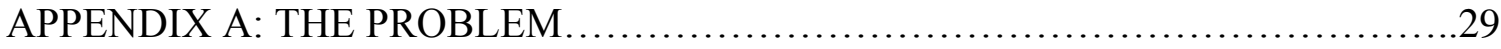

APPENDIX B: LITERATURE REVIEW .........................................38

APPENDIX C: ADDITIONAL METHODS ......................................65

APPENDIX D: ADDITIONAL RESULTS.........................................72

APPENDIX E: RECOMMENDATION FOR FUTURE RESEARCH.................76

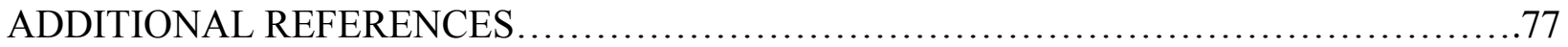




\section{LIST OF TABLES}

Table B1.DIII Games/Athletes per Game/Practices/Athletes per Practice NCAA Sports........39

Table B2. DIII Preseason Games and Practices with Associated Injury Rates by NCAA.......40

Table B3. DIII In Season Games and Practices with Associated Injury Rate by NCAA.........41

Table B4. DIII Post Season Games and Practices with Associated Injury Rate by NCAA......42

Table B5. DIII Total Games and Practices with Associated Injury Rates by NCAA............43

Table B6. Percentage of Game and Practice Injuries Upper Extremity.....................44

Table B7. Percentage of Game and Practice Injuries Lower Extremity ......................45

Table B8. Percent of Injuries that Required 10+ Days of Time Loss.......................45

Table C1. Consent Information for the Athlete.......................................65

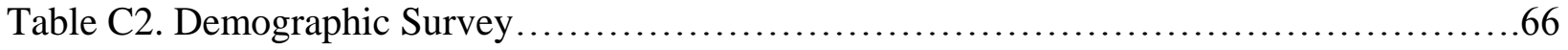

Table C3. Rehabilitation Over Adherence Questionnaire (ROAQ).........................67

Table C4. Athletic Identity Measurement Scale (AIMS) ................................68

Table C5. Consent Information for the Athletic Trainer.................................69

Table C6. Sport Injury Rehabilitation Adherence Scale (SIRAS) ..........................70

Table C7. Rehabilitation Adherence Measure for Athletic Training (RAdMAT)...............71

Table D1. Demographic Information............................................. 72

Table D2. Repeated Measures ANOVA 1x2 ROAQ, SIRAS, and RAdMAT ..................73

Table D3. Correlation between Athletic Identity and ROAQ...............................73

Table D4. Correlation Analysis for AIMS \& Years of Participation.........................73

Table D5. Correlation Analysis for SIRAS \& RAdMAT ..................................73 


\section{LIST OF FIGURES}

Figure D1. Sport Participation....................................................... 74

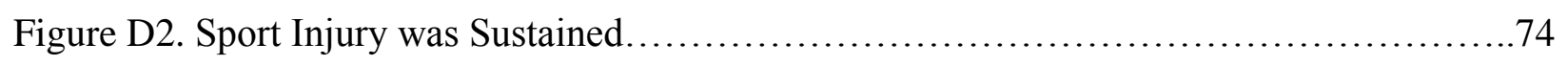

Figure D3. Years of Sport Participation......................................... 75 


\section{INTRODUCTION}

During the 2002-2003 academic school year, a reported 450,000 athletes participated in collegiate athletics representing the five major collegiate associations. ${ }^{1}$ Of these 450,000 athletes, 137,000 represented the Division III (DIII) level. ${ }^{1}$ At the Division III level there was a reported 350,203 athlete exposures with males representing $62.6 \%$ of exposures. ${ }^{1}$ A reported 8,439 injuries occurred at the Division III level with males accounting for $60.3 \%$ of injuries. ${ }^{1,2}$ Some of those resulted in time loss or non-time loss injury. A time loss injury requires an evaluation being performed by a certified athletic trainer and required the athlete to be withheld from sport. ${ }^{1,2}$ Time loss injuries were more prevalent with males, while females experienced more non-time loss injuries as more males compete in collision and contact sports while females are more likely to compete in non-contact sports. ${ }^{1,2}$ Non-time loss injuries also required an evaluation, but ultimately does not require restriction from sport. ${ }^{1-3}$ Time loss injuries are more acute in nature compared to non-time loss injuries, which are more chronic in nature. ${ }^{1-3}$ With non-time loss injuries being more chronic in nature, athletes maybe returning to sport too quickly or playing through a chronic injury. ${ }^{1-3}$ For men's sports wrestling, basketball, football, and soccer reported the highest prevalence for non-time loss injuries, and football, soccer, wrestling, and basketball comprised the highest number of time loss injuries. ${ }^{1-4}$ For women's athletics, volleyball, basketball, soccer, and field hockey represented the most non-time loss injuries and soccer, field hockey, and basketball represented the highest rates of time loss injuries. ${ }^{1-4}$

There were a reported 70,361 injury treatments provided at the Division III level with $55.4 \%$ of treatments being performed on male athletes. ${ }^{1,2}$ Male sports averaged 7.7 treatments per injury, with 6.1 treatments performed for non-time loss injuries and 11.8 treatments for time 
loss injuries. ${ }^{1}$ Women reported receiving more treatments with an average of 9.4 treatments per injury, 7.3 treatments for non-time loss injuries, and 18.2 treatments for time loss injuries. ${ }^{1}$ Athletes who suffered from time loss injuries received more treatments compared to athletes who sustained a non-time loss injury. Athletes with non-time loss injuries experience pain, but can manage the pain so that it has minimal effects on performance.

At this time the National Athletic Training Association (NATA) has a reported 23,000 certified athletic trainers with $20 \%$ of these athletic trainers working in the college or university setting. ${ }^{1}$ Athletic trainers are the first line of defense for the medical team and see athletes through the rehabilitation process whether for a time loss or non-time loss injury. ${ }^{1-4}$ Unfortunately, for the athletic trainer, not all are prepared to determine which athletes will be adherent or not to a rehabilitation program. ${ }^{1-4}$ Adherence levels ultimately affects return to play status. ${ }^{5}$ Knowing and understanding epidemiology helps shape theoretical frameworks aimed at enhancing knowledge regarding adherence to sport injury rehabilitation.

Prior to the development of theoretical frameworks, the research that was conducted was atheoretical. ${ }^{5}$ These frameworks helped to enhance the knowledge of sport injury adherence. ${ }^{5-16}$ The theoretical frameworks allowed for variables to be studied, research questions to be formulated, and to project experimental hypotheses. ${ }^{5-16}$ The reported literature has helped to develop and apply numerous theoretical frameworks for adherence in rehabilitation programs to enhance understanding. ${ }^{5-16}$ Studies have been conducted applying components of these theories demonstrating some support of these frameworks. ${ }^{5-16}$ Commonly discussed frameworks in the literature include personal investment theory (PIT),${ }^{5-11}$ protection motivation theory (PMT),${ }^{5-10}$ cognitive appraisal models, ${ }^{5,12-14}$ attribution theory, ${ }^{5}$ theory of planned behavior (TPB), ${ }^{5,7,16}$ and health action process approach (HAPA). ${ }^{5,7,15}$ 
Understanding epidemiology of sport injuries, access to certified athletic trainers, and theoretical frameworks' regarding sport injury adherence levels is important in understanding the concept of adherence. As clinical outcomes determine return to play activity, and adherence affects clinical outcomes, how to determine adherence is an on-going science. There is a lack of a consistent definition regarding adherence, however, a commonly used definition is, "an active, voluntary collaborate involvement of the patient in a mutually acceptable course of behavior to produce a desired preventative or therapeutic result." ${ }^{\text {17 }}$ Due to the lack of a unified definition, there is no gold standard reported for measuring adherence. ${ }^{18}$ As over 200 variables can affect adherence, a few reported factors that can influence adherence levels include injury severity, gender, sport type, level of competition, scholarship status, and playtime. ${ }^{19,20}$ Examples of assessments for adherence include the Sport Injury Rehabilitation Adherence Scale (SIRAS), ${ }^{18,21-}$ ${ }^{24}$ Rehabilitation Adherence Questionnaire (RAQ) ${ }^{25}$ the Rehabilitation Over Adherence Questionnaire (ROAQ) $)^{13}$ and the Rehabilitation Adherence Measure for Athletic Training (RAdMAT) ${ }^{18}$ The lack of a definition and standardized measurement of adherence leads to a lack of clear guidelines for what constitutes under adherent, adherent, and over adherent behavior during the rehabilitation process..$^{20,26,27}$

Athletic trainers have reported that under adherence is a frequent issue experienced in clinical practice, and over adherence is an issue in clinical practice occasionally. ${ }^{20}$ Thus, most of the literature has focused on under adherent behavior with little research focusing on over adherent behavior. ${ }^{13,20}$ It has been stated that in clinical based rehabilitation adherence rates ranged from $40-91 \%$ thus indicating the need to further study adherence to sport injury rehabilitation in an athletic training setting. ${ }^{18,20,26,27}$ Both under and over adherence cause delays in return to participation. ${ }^{13,20}$ 
Injuries that are more severe in nature tend to result in loss of playtime and tend to have a longer rehabilitation, hence referred to as time loss injuries. ${ }^{20}$ Time loss injuries are more prevalent in collision and contact sports while non-time loss injuries are more prevalent in noncontact sports. ${ }^{1}$ Due to the high velocity and impact nature of collision and contact sports participating athletes are placed at a higher risk for injury. Thus, football, soccer, wrestling, and basketball comprised the highest number of time loss injuries. ${ }^{1}$ As males are more likely to participate in collision and contact sports and females are more likely to participate in noncontact sports males may then experience more time loss injuries, while females experience more non-time loss injuries. ${ }^{1}$ Time loss injuries have a negative effect on adherence levels because the implementation back to sport is not within the immediate future. ${ }^{20}$ Athletes who have sustained a time loss injury may have a hard time maintaining motivation during rehabilitation sessions resulting in under adherent behavior. ${ }^{20}$ In contrast, athletes may believe that increasing the frequency of rehabilitation exercises repetitions, sets, and weight will result in an expedited return to play, thus leading to over adherent behavior. ${ }^{13}$ However, this can lead to further injury delaying implementation back to sport. ${ }^{13}$

It has been stated that achieving optimal adherence levels at the Division III level was a challenge. ${ }^{20}$ Under adherence has been an issue experienced in the physical therapy and athletic training clinical settings especially at the Division III level of competition. ${ }^{13,18-20,27}$ These low levels of adherence may be related to ignoring practitioner recommendations, not attempting an expedited return, or that theoretical frameworks such as the cognitive appraisal model, personal motivational theory, and theory of planned behavior are not being used to help enhance adherence to sport injury rehabilitation. ${ }^{5-10,12-14,16}$ As long as athletes are competing in NCAA sanction sports time loss and non-time loss injuries will be prevalent and can lead to over or 
under adherence. Athletic trainers are the first line of defense in treating an athlete and see the athlete through the rehabilitation process. Athletic trainers are able to evaluate good effort, ability to follow clinicians' instructions and the receptiveness of the athlete to changes to the rehabilitation protocols. Therefore, evaluating athlete and athletic trainer perceptions concerning adherence is of vital importance in order to achieve optimal clinical outcomes. Further, very few studies have evaluated adherence levels by including both the athlete and athletic trainer. As little reported literature exists on compliance at the DIII level, the purpose of this study was to evaluate adherence levels at the DIII level comparing adherence scores through a pre and posttest.

\section{METHODS}

Design

This was a prospective exploratory study that included data collected from five written questionnaires. Athlete participants completed a demographic questionnaire, a 7-item athletic identity measurement scale (AIMS), and a 10-item Rehabilitation Over Adherence Questionnaire (ROAQ). Athletic trainer participants completed the 3-item Sport Injury Rehabilitation Adherence Scale (SIRAS) and the16-item Rehabilitation Adherence Measure for Athletic Training (RAdMAT).

Subjects

Athlete participants $(\mathrm{n}=12)$ in this study were athletes who had sustained a musculoskeletal injury participating in NCAA sanctioned sports at one Division III institution. Athletes reported being 18-22 years old and participating in football, soccer, baseball, volleyball, indoor track, and outdoor track. Inclusion criteria included sustaining a musculoskeletal injury resulting in withdrawal from sport participation for ten or more days in order to complete 
rehabilitative activities. Athletes were at least 18 years of age or older, with a physical on file, be a member of a sport team, and participating in practices and/or games prior to injury. Participants were expected to fill out the appropriate questionnaire at the conclusion of the rehabilitation session. Any individual not participating in collegiate athletics at this Division III institution was excluded from the study.

Three athletic trainers participated in the study. Inclusion criteria for athletic trainers included being a practicing licensed athletic trainer at this Division III institution and work with team and individual sport athletes. Exclusion criteria for athletic trainers included not being licensed in the state of Pennsylvania and not working with sport teams or individual athletes at this Division III institution. This study was approved by the Institutional Review Board (IRB) for protection of human subjects at two institutions. Instrumentation

Instruments utilized in this study included the 3-item Sports Injury Rehabilitation Adherence Scale (SIRAS), ${ }^{18,21-24} 10$ - item Rehabilitation Over Adherence Questionnaire (ROAQ), ${ }^{13}$ 16-item Rehabilitation Adherence Measure for Athletic Training (RAdMAT), ${ }^{18}$ the 7-item Athletic Identity Measurement Scale (AIMS) $)^{13,33,34}$ and a demographic survey developed by the primary investigator.

The 3-item SIRAS has been utilized in the sports medicine clinic. ${ }^{18,21-24}$ This measure is completed by the health care professional. ${ }^{18,21-24}$ The three items address the patients' intensity, frequency, and receptiveness to instruction provided by the health care professional ${ }^{18,21-24}$ A 5point Likert scale is used with 1 representing minimum effort, never, and very unreceptive and five representing maximum effort, always, and very receptive. ${ }^{18,21-24}$ 
In 2002, Brewer ${ }^{21}$ conducted a study to determine construct validity and interrater agreement. Rehabilitation practitioners of various experience watched and evaluated hypothetical videotapes of patients demonstrating low, moderate, and high adherence. ${ }^{21}$ Scores for highly adherent $(M=14, S D=1.27)$ were substantially higher than moderate scores $(M=8.93$, $\mathrm{SD}=1.67)$, and moderate scores were significantly higher than lowly adherent scores $(M=4.79$, $\mathrm{SD}=.93){ }^{21}$ This study showed that the rater-agreement index (RAI) ranged from .84 for low adherence, .86 for moderate, and .90 for high with an average value of $.87 .{ }^{21}$ These statistics represent a high level of interrater agreement for the SIRAS. ${ }^{21}$ Continuing this study, Brewer ${ }^{21}$ addressed the limitation of hypothetical scenarios. Two athletic trainers completed the SIRAS independently using twelve patients with anterior cruciate ligament reconstruction over the course of four rehabilitation sessions. ${ }^{21}$ The RAI value for the four sessions was .94 thus demonstrating a high level of interrater agreement for the SIRAS. ${ }^{21}$ The results from this study state that SIRAS scores will be similar for different practitioners observing the same patient in the clinical setting. ${ }^{21}$

The 10 -item ROAQ is newly reported in the literature. ${ }^{13}$ This measure evaluated an athlete's likelihood of engaging in behaviors that are defined to be over adherent. ${ }^{13}$ The ROAQ includes 10-items and uses a 5-point Likert scale with one representing never and five representing always. ${ }^{13}$ The subscales that are used in this measure included ignoring practitioner recommendations and an expedited rehabilitation. ${ }^{13}$ In the literature there is no measurement that addressed over adherent behavior in a rehabilitation program. ${ }^{13}$ Therefore, this measure was developed utilizing the author's knowledge on the content, clinical experience, and research regarding the topic. ${ }^{13}$ 
Podlog ${ }^{13}$ conducted a studying implementing the use of the ROAQ in an adolescent population. The study concluded that athletic identity was positively correlated to the two subscales of the ROAQ for ignoring practitioner guidelines $(r=.23)$ and attempting an expedited return $(r=.46) .{ }^{13}$ The study population reported moderate levels of willingness to make a premature return to sport with a value of $3.30 .{ }^{13}$ The adolescents study reported moderate to low levels of over adherence with values of 2.01 for ignoring practitioner recommendations and 3.11 for attempting an expedited return. ${ }^{13}$ To elaborate on the population, Podlog ${ }^{13}$ conducted the study with a collegiate sample. In this study Cronbach's alpha coefficients for the two subscales of 0.86 and 0.75 indicated acceptable internal reliability. ${ }^{13}$ The major conclusion from the two studies indicated that the ROAQ has adequate construct validity and internal reliability. ${ }^{13}$ The ROAQ can be administered in research and rehabilitative settings. With athlete consent, the ROAQ can be used as a teaching instrument educating the patient on the negative clinical outcomes that can occur due to over adherent behavior. ${ }^{13}$

The 16-item RAdMAT was completed by the athletic trainer. ${ }^{18}$ Three subscales of attendance/ participation, communication, and attitude/effort are included ${ }^{18} \mathrm{~A}$ four point Likert scale ranged from one representing never, two occasionally, three often, and four always. ${ }^{18}$ High internal consistency along with positive and strong relationship between the RAdMAT total and SIRAS total have been noted. ${ }^{18}$ The internal consistency Cronbach's alpha values that were reported for least, average, and most adherent athlete ranged from .748-.919 indicating an acceptable range. ${ }^{18}$ Correlation analyses reported strong, positive, and significant relationships between the RAdMAT and SIRAS subscales. ${ }^{18}$ The SIRAS is an accepted and strong measure for adherence in a sports medicine clinic. ${ }^{18,20}$ The RAdMAT and SIRAS were developed for the 
athletic training setting to determine between group differences. ${ }^{18,20}$ However, the SIRAS may be preferred since it is a short measure utilized over many sessions. ${ }^{18,20}$

The AIMS is a 7-item questionnaire that evaluates how strongly an athlete identifies with the athletic role. ${ }^{13,33,34}$ The three subscales utilized in this measure include social identity, exclusivity, and negative affectivity. ${ }^{13,33,34}$ A 7-point Likert scale is used with one representing strongly disagree and seven representing strongly agree..$^{3,33,34}$ This test has reported validity and reliability within an adolescent athletic population. ${ }^{13}$ In addition to high test-retest reliability (0.89), internal consistency (0.80-0.93), concurrent validity, and construct validity are also evident. $^{13}$

Finally, a demographic questionnaire was developed by the primary investigator in order to collect information regarding sex, age, sport, education status, and injury. The selections consisted of forced choice questions as well as fill in the blanks.

Procedures

Participants who are athletes at the Division III institution whom meet the inclusion criteria were approached to participate in the study. The purpose of the study was explained to the participants before reading the cover letter (Table C1). If participants agreed to participate than the demographic questionnaire (Table C2), ROAQ (Table C3), and AIMS (Table C4) were completed following the rehabilitation session. The ROAQ was completed as a pre-test at the first data collection and a post-test at the second data collection or at the conclusion of a threeweek rehabilitation program.

Participants who are licensed certified athletic trainers at the Division III institution whom met inclusion criteria were approached to participate in the study. The purpose of the study was explained to the participants before reading the cover letter for the athletic trainer 
(Table C5. After, agreement to participate, athletic trainers completed the SIRAS (Table C6) and the RAdMAT (Table C7) following rehabilitation sessions as a pre and post-test.

Determinants to remain in the study included completion of the questionnaires.

Data Analysis

A point value for the SIRAS (Table C6) was determined by adding the numerical results from the three items. ${ }^{18,21-24}$ Scores can range from three to fifteen, with scores nearing fifteen representing high adherence, scores of eight representing moderate adherence, and scores nearing three representing low levels of adherence. ${ }^{18,21-24}$ The ROAQ (Table C3) is scored by adding the numerical totals from the ten items. ${ }^{13}$ Scores can range from ten to fifty, with scores nearing fifty being indicative of overadherence, and scores nearing ten indicating adherence or under adherence. ${ }^{13}$ The RAdMAT (Table C7) is scored by adding the values from the 16items. ${ }^{18}$ Scores can range from sixteen to sixty-four, with scores nearing sixty-four representing most adherent, scores nearing thirty-two as average adherence, and scores nearing sixteen as least adherent. ${ }^{18}$ Finally, the AIMS (Table C4) is calculated by adding the numerical totals from the seven items. ${ }^{13,33}$, Scores can range from seven to forty-nine, with scores nearing seven as low level of athletic identity, scores nearing twenty-five as moderate athletic identity, and a score of forty-nine indicating high levels of athletic identity. ${ }^{13,33,34}$

Statistical Analysis

The independent variable that was studied was time. The dependent variable was adherence. Descriptive analysis utilized mean percentages and frequencies for athlete responses based on demographic information and athletic identity responses. Originally, statistical analysis included a 2 x 3 way repeated measures ANOVA (sport $x$ time) to compare the score for the ROAQ between sports over three time points. Another 2 × 3 repeated measures ANOVA 
(gender X time) was to be utilized. However, due to the lack of variability of gender and sports within the participants, $1 \times 2$ repeated measures ANOVA was utilized to determine differences between pre and post-tests.

Originally, statistical analyses used for athletic trainers included a 2 x 3 repeated measures ANOVA (sport $\mathrm{x}$ time) as well as (gender $\mathrm{x}$ time) in order to compare scores from the SIRAS and the RAdMAT. Team and individual sports and males and females were evaluated over three time points. However, due to the lack of variability in participants, a 1x2 repeated measures ANOVA was utilized to determine differences in scores over the course of pre and at the conclusion of a three-week rehabilitation program or at the end of a rehabilitation program.

Correlation statistics for the athlete were utilized to compare scores from the AIMS and the ROAQ in order to see if there is a relationship between athletic identity and adherence scores. An additional correlation was utilized to compare scores from the AIMS and years of athletic participation to see if there is a relationship between athletic identity and years of sport participation. Correlation statistics for athletic trainer responses was utilized for the SIRAS and the RAdMAT to see if there was a relationship between the two test measures. A probability level of $\mathrm{p}<0.05$ was set for all tests. All statistical analyses were conducted using SPSS for Windows (version 21.0; SPSS, Inc. Chicago, IL).

\section{RESULTS}

Demographic Results

Of the twelve demographic survey's completed, the majority of the distribution were male participants $(91.7 \% \mathrm{n}=11)$ compared to female $(8.3 \% \mathrm{n}=1)$ participants. All participants' ages were $18-22(100 \% \mathrm{n}=12)$. The sports that were represented included football $(58.3 \% \mathrm{n}=7)$, soccer $(16.7 \% \mathrm{n}=2)$, baseball $(8.3 \% \mathrm{n}=1)$, and multiple sports $(16,7 \% \mathrm{n}=2)$. The numbers of 
injuries per represented sport were football $(58.3 \% \mathrm{n}=7)$, soccer $(16.7 \% \mathrm{n}=2)$, indoor track $(8.3 \%$ $\mathrm{n}=1)$, baseball $(8.3 \%, \mathrm{n}=1)$, and intramurals $(8.3 \% \mathrm{n}=1)$. The years of sport participation were $0-5$ years $(16.7 \% \mathrm{n}=2), 6-10(8.3 \% \mathrm{n}=1), 10-15(41.7 \% \mathrm{n}=5)$, and 15 or more $(33.3 \% \mathrm{n}=4)$. The subjects were split with the distribution of history of injury with yes $(50 \% \mathrm{n}=6)$ and no $(50 \%$ $\mathrm{n}=6)$. The class status varied with freshmen $(41.7 \%, \mathrm{n}=5)$, sophomore $(33.3 \%, \mathrm{n}=4)$, and junior $(25 \%, \mathrm{n}=3)$. The season when athletes sustained the injury was in-season $(83.3 \% \mathrm{n}=10)$ and post-season $(16.7 \% \mathrm{n}=2)$. Refer to Table D1.

Athlete Results

A 1x2 repeated measures ANOVA was utilized with time being the factor for a pre and post-test. Three participants did not complete the post-test. The repeated measures ANOVA was completed without the data and then using intention to treat. Intention to treat includes subjects that started the study were assumed to complete the study as if the pre and post were completed. There was no significant difference, so statistical analysis included the intention to treat. The ROAQ, which assessed the likelihood of an athlete engaging in over adherent behavior was assessed. There were no significant differences between pre and post-test scores for the ROAQ $(\mathrm{F}=2.382, \mathrm{P}=0.151)$. The mean score on the ROAQ pre-test was 19.416, indicating that DIII athletes in the study are not likely to engage in behaviors that are classified as over adherent. Similar results for the ROAQ post-test were also found with a mean score of 21. Scores for this measure ranged from 12-36 for the pretest and 13-32 for the post-test. Refer to Table D2.

A correlation was run to assess if there was a relationship between athletic identity and over adherent behavior. The Pearson correlation revealed that there was a weak to moderate correlation comparing the pre $(\mathrm{r}=.314 \mathrm{p}=.320)$ and post-test $(\mathrm{r}=.319 \mathrm{p}=.313)$ for the ROAQ. The 
mean score on the athletic identity score was 40.916 out a total of 49 . The range for the AIMS was 26-49. This is indicative that the majority of DIII athletes in the study reported high levels of athletic identity through the AIMS. An additional correlation was conducted in order to evaluate if there was a difference between athletic identity and years of sport participation $(\mathrm{r}=.354 \mathrm{P}=.261)$. This correlation revealed a non-significant moderate correlation between athletic identity and years of participation. Refer to Table D3.

Athletic Trainer Results

Two separate $1 \times 2$ repeated measures ANOVA with time as the factor for a pre and posttest was utilized. Three participants did not complete the post-test. The repeated measures ANOVA was completed without the three participants, and then again with the intention to treat. Again, there was no significant difference, so statistical analysis included the intention to treat. For the SIRAS there were no significant differences $(\mathrm{F}=.786, \mathrm{P}=.394)$ between pre and post-test scores. The mean score that was reported on the SIRAS pre-test was 13.08 signifying good effort, ability to follow clinicians instructions, and being receptive to the clinicians changes to the rehabilitation protocol. Similar findings were found with the post-test for the SIRAS with the mean score being 13.41. The range for pre-test scores was seven to fifteen and eight to fifteen for the post-test. For the RAdMAT there was no significant difference $(\mathrm{F}=.592, \mathrm{P}=0.458)$ between the pre and post-test scores. The mean score for the RAdMAT pre-test was 54 out of 64 representing moderate to high levels of adherence. Similar results were found for the posttest with a mean score of 55.66 also representing moderate to high levels of adherence. The range for the pre-test was thirty-three to sixty-four and for the post-test forty-five to sixty-four Refer to Table D2. 
A correlation was utilized to evaluate if there was a relationship between the SIRAS and the RAdMAT. There was a strong, significant correlation between the pre SIRAS and pre RAdMAT $(r=.932 \mathrm{p}>0.01)$, post SIRAS and pre RAdMAT $(\mathrm{r}=0.764 \mathrm{p}=0.004)$, pre SIRAS and post RAdMAT ( $\mathrm{r}=.714 \mathrm{p}=.009)$, post SIRAS and post RAdMAT $(\mathrm{r}=.673 \mathrm{p}=.016)$, and SIRAS total and RAdMAT total $(\mathrm{r}=0.901 \mathrm{p}>0.001)$.

\section{DISCUSSION}

The purpose of this study was to evaluate adherence levels to sport injury rehabilitation at the DIII level over the course of a three-week period. The aim of this study was also to provide additional research regarding adherence at the Division III level. In this study, athletes and athletic trainers completed various questionnaires assessing athlete's adherence to sport injury rehabilitation. From this study it was found that the athletes at the Division III level were not over adherent even though high levels of athletic identity were displayed. In addition, the athletic trainers reported that the athletes were moderately to highly adherent. These results do not support what has been reported in the literature regarding adherence at the Division III level. The literature has stated that under adherence has been an issue experienced in the physical therapy and athletic training clinical settings especially at the Division III level of competition. ${ }^{13,18-20,27}$ Athletic trainers have also commented that over adherent behavior is an issue occasionally experienced in clinical practice. ${ }^{13,20}$ In this study, neither over or under adherence was apparent.

The results from the questionnaires highlighted that athletes competing at the Division III level are not likely to over adhere to the rehabilitation protocol. ${ }^{13,20}$ The athletes in this study also displayed high levels of athletic identity meaning that the athletes highly identify with the athletic role. The results from this study exemplify that athletic identity and over adherent 
behavior are weakly to moderately correlated indicating that these D III athletes at this institution were not likely to over adhere even though high levels of athletic identity were displayed. This could mean athletes are cognizant that engaging in sport specific activities too soon or increasing sets, repetitions, and weight too quickly may have detrimental effects regarding return to play outcomes. In addition, this could be representative of positive rapports that athletes have with athletic trainers. Athletic trainers may be better educating patients on the negative clinical outcomes that can be present regarding under and over adherence to the prescribed rehabilitation program.

The relationship between years of sport participation and athletic identity was also explored. Through the correlation it was found that there is not a significant relationship between sport participation and athletic identity meaning that athletes who only competed for zero to five years could demonstrate similar athletic identity scores for an athlete with fifteen years or more experience with sport participation.

\section{Athlete Adherence}

The literature reports over 200 variables that can affect adherence levels. ${ }^{19,20,27}$ Some of these variables include injury, severity, age, competitive level, prior history of injury, sport type, and gender. ${ }^{19,20,27}$ In this study, the definition of an injury was the definition of severe injury as stated by the NCAA, which is an injury resulting in ten or more days lost from participation.,3544 The injury and severity have been reported variables to affect adherence rates. ${ }^{19,20,27,}$ It has been projected that athletes with longer rehabilitation rates display decreased adherence rates since return to sport is far removed. ${ }^{19,20,47}$ Injuries requiring surgical interventions may have a longer projected return to play compared to an injury that does not require a surgical intervention. Sustaining a season ending injury requiring surgery is psychologically challenging 
for athletes. Athletes may experience more drastic changes in adherence following the first two weeks of injury. In this study, there were athletes that required surgical and nonsurgical interventions. The surgical athletes, mostly ACL surgeries, were in the middle to end phase of the protocol, while those of a non-surgical nature were more recent. It was noted that those surgical rehabilitation cases were adherent and very compliant, perhaps that these athletes were nearing the end of the rehabilitation. It is questioned whether adherence would have been different if the athletes that required a season ending surgery were followed from immediate post-surgical rehabilitation to discharge from rehabilitation to return to sport. This would have provided a different perspective to adherence and perhaps a more realistic picture of a DIII athlete following a time loss injury, rather than at the end of the rehabilitation protocol.

It has been noted in the literature that the type of sport and the gender of the athlete in those sports affects adherence levels. ${ }^{19,20,47}$ The majority of the sample was collision sport athletes followed by contact, and noncontact. Collision sports involve athletes purposely hitting one another. Contact sports entail a range of contact between athletes, ground, or ball. Noncontact sports include no physical contact happening between players. Due to the high velocity and impact nature of collision sports these athletes are more prone to sustaining a time loss injury. Males are more likely to participate in collision and contact sports while females are more likely to participate in non-contact sports. ${ }^{1}$ Football, soccer, wrestling, and basketball athletes have reported sustaining the highest number of time loss injuries. ${ }^{1}$ This study supports the information stated in the literature. The majority of the sample were males $(n=11)$ who sustained a time loss injury participating in collision or contact sports (football $\mathrm{n}=7$ and soccer $\mathrm{n}=2$ ). The only female present in the study sustained her injury during indoor track season. The football and soccer athletes sustained injuries that required a significant time loss from sport 
with a surgical intervention. Depending on the severity of the injury will determine a general timeline of cessation from activity, which could be indicative of decreased adherence rates. However, this was not the case as all time-loss injuries did not affect adherence with athletes in this study. All athletes were adherent. As seven of the 11 athletes played football, it was assumed that these individuals would be the least adherent as decreased adherence rates for football athletes have been reported in the literature. ${ }^{20}$ Again decreased adherence was not evident in this study. As this patient population was not representative of all D III athletes, future research should focus on adherence levels that males and females demonstrate on sport teams at the D III level and compare male and female adherence scores.

Elite athletes are more likely to report higher athletic identity scores compared to recreational athletes. ${ }^{34}$ The literature stated that athletes who report higher levels of athletic identity are more likely to participate in sport. ${ }^{34}$ Athletes who report high levels of athletic identity are at an increased risk of demonstrating over adherent behavior, which includes ignoring practitioner recommendations and attempt an expedited return to play. In this study, athletes reported high levels of athletic identity, however, they did not demonstrate over adherent behavior. This could be due to the fact that half of the sample had experienced a prior injury and was familiar with sport injury rehabilitation. In addition, this finding could be indicative of the positive relationship between athletes and athletic trainers at this D III institution. Athletic trainers can be educating patients that under engaging and over engaging in the rehabilitation protocol can both have detrimental effects on clinical outcomes and could delay return to play. Athletic Trainer

The athletic trainer literature in evaluating the injured athletes' adherence to rehabilitation protocol is scarce and even scarcer in relation to the Division III setting. The 
results from this study regarding athletic trainers' evaluation of injured athletes' adherence to the rehabilitation protocol conflicts with the reported literature. Most of the literature focuses on evaluating adherence in the clinical setting, not in the athletic training setting. ${ }^{18-20,27}$ Of the studies that are conducted utilizing the athletic training setting, athletic trainers have expressed issues with experiencing optimal adherence at the Division III level especially with football athletes. ${ }^{20}$ No research has focused on over adherent behavior at the Division III level. Under and over adherence are issues that can be experienced in clinical practice, which can both lead to detrimental clinical outcomes and delayed return to play. Athletic trainers reported through the pre and post-test using the SIRAS and RAdMAT that the athletes demonstrated moderate to high levels of adherence to the prescribed rehabilitation program. Using SIRAS results, it was reported that the athletes put in a good effort, followed clinicians' instructions, and were receptive to the clinicians' changes to the rehabilitation protocol. Similar findings were found with the post-test for the SIRAS.

The SIRAS and RAdMAT provide a means for the athletic trainer to evaluate an athlete's progress other than completing a rehabilitation summary sheet. The SIRAS provides information about the athlete's intensity, frequency and receptiveness to the instruction provided by the athletic trainer. ${ }^{18,21-24}$ The RAdMAT also provides similar information and reports on attendance/participation, communication and attitude/effort. ${ }^{18}$ By using these two instruments, adherence can be more adequately followed throughout the rehabilitation period, especially since athletes in the athletic training setting may be coming in for treatment at least two times a day, throughout the week and perhaps weekends. Due to the accessibility of the athletic training room to athletes and individualized athlete-athletic trainer interaction it could be a concern that an over adherent environment could be fostered. Simply completing the two or the SIRAS would 
be beneficial after attainment of protocol milestones to note if under, adherence or over adherence is evident to plan the rehabilitation accordingly. It has been shown that the relationship between the SIRAS and the RAdMAT is a positive, strong relationship as noted in the existing literature. This finding also enforces that the SIRAS and RAdMAT will yield similar results at the Division III level as was noted in this study.

Theoretical Framework Application

Theoretical frameworks have been utilized to enhance the knowledge concerning adherence to sport injury rehabilitation. Frameworks that have been discussed in the literature include personal investment theory (PIT), ${ }^{5-11}$ protection motivational theory (PMT), ${ }^{5-10}$ cognitive appraisal models, ${ }^{5,12-14}$ attribution theory, ${ }^{5}$ theory of planned behavior (TPB), ${ }^{5,7,16}$ and health action process approach (HAPA).$^{5,7,15}$ Of the six theoretical frameworks discussed, the three that are most applicable to sport injury rehabilitation includes cognitive appraisal models, PMT, and TPB.

The Wiese-Bjornstal et al. model ${ }^{5,12-14}$ is the most commonly utilized cognitive appraisal model. With a focus on post-injury behavior, this model is a continuation of the Williams and Anderson model focusing on pre-injury behavior., ${ }^{5,12-14}$ Personal and situational factors affect how an athlete will cognitively appraise an injury. ${ }^{5,12-14}$ The cognitive appraisal will depend on various factors such as the meaning of the injury to the athlete, consequences of the injury, and the athletes ability to cope with the injury. ${ }^{5,12-14}$ Cognitive appraisals can be either positive or negative and affect emotional and behavioral responses., ${ }^{512-14}$ This content applies to the present study because the ROAQ focused on a behavioral response concerning adherence to a sport injury. An athlete's emotional response to injury can affect the behavioral response of ignoring the practitioner's guidelines concerning repetitions, sets, and weight progress for therapeutic 
exercises in order to have an expedited return to play. The time of season when the injury occurs can affect how an athlete cognitively appraises the injury. In this study the majority of injuries occurred during the competitive season or in the post-season. Athletes may initially experience decreased adherence levels with an injury that requires a cessation from sport participation during the competitive season. In addition, athletes may experience decreased adherence rates with injuries occurring during the off-season due to the fact that the competitive season is far away. Why the athletes' behavior did not result in over and under adherence may perhaps be related to the positive rapport evident between the athlete and the athletic trainer or that the athletes, despite having a season ending injury and in the off-season, were nearing the end of the rehabilitation. As this study did not focus on an athlete's emotional and behavioral response post-injury but rather adherence, future research should focus on those two aspects to better understand the athletes experience following a sport injury.

Another model that has been utilized in the literature and applies to the current study includes PMT. The PMT is a continuation of the health belief model. ${ }^{10}$ It is premised that it is an individuals desire to protect themselves from a health aliment. ${ }^{5-10}$ The individuals ability to protect themselves depends on how serious or how likely the perception of sustaining the aliment. ${ }^{5-10}$ The ability to overcome the aliment is determined by the belief in treatment effectiveness and the ability to engage in activities that will help improve health status. ${ }^{5-10}$ In this theory there are two cognitive appraisals that take place they include threat and coping appraisals. ${ }^{5-10}$ Threat appraisals are classified as the patients perceived severity and susceptibility. ${ }^{5-10}$ Coping appraisals occur when the individual realized the positive effects of rehabilitation and ability to perform exercises. ${ }^{5-10}$ Taylor and $\mathrm{May}^{8}$ conducted a study and stated that injury severity and susceptibility led to decreased adherence rates while self-efficacy and 
belief in treatment caused increase in adherence. In the present study, athletes had sustained a severe injury and displayed moderate to high adherence rates. The athletes were in the mid to end phases of the rehabilitation beginning to perform functional exercises. Athletes were able to successfully complete rehabilitation exercises assigned by the clinician promoting self-efficacy. Due to the athlete-athletic trainer rapport, athletes may have higher beliefs in treatment as a relationship is built. It is important for athletic trainers to educate athletes concerning the importance of rehabilitative exercises and modalities to the patient in order to promote belief in treatment. In addition to educating the athlete that susceptibility and severity could become more prevalent by not adhering to clinician instructions.

The final theoretical framework that has been discussed in the literature is the TPB. This theory is a continuation of the theory of reasonable behavior. ${ }^{5,7,16}$ The TPB has been applied to adherence to sport and included athletes intentional planning to complete or not complete rehabilitative exercises. ${ }^{5,7,16}$ Intentional planning is affected by attitudes toward the behavior, subjective norms, and perceived behavioral control. ${ }^{5,7,16}$ The stronger the intention of performing rehabilitation exercises the more likely an athlete is to do so. ${ }^{57.16}$ Factors such as money, time, skill, and accessibility can deter an athlete from completing rehabilitation exercises. ${ }^{5,7,16}$ How an athlete perceives the ability to perform an activity determines how likely the athlete is to actually perform the activity, and can influence adherence. ${ }^{5,716}$ The theory described above is applicable to the current study due to the environment of the athletic training room. Although time constraints can be a deterrent for student athletes to go to treatment, athletic trainers try to plan treatment times around athlete's class and practice schedule in the attempt to provide an intentional time allowing the athletes to perform rehabilitation exercises. Clinical Implications 
Results from this study suggested that adherence to sport injury rehabilitation does not change over the course of a three week period indicating that athletes will display the same level of adherence during the course of the rehabilitation protocol. This can help athletic trainers pinpoint which athletes will require various motivational techniques to improve return to play outcomes. The athlete and athletic trainer relationship allows athletic trainers a unique position to discuss these factors with the athlete. Athletes are more likely and willing to receive medical advice from the athletic trainer compared to the coaching staff, teammates, and family. In addition, due to the time spent in the athletic training room, athletic trainers provide social support and assistance regarding emotional, tangible, and informational support, to the athletes during sport injury rehabilitation. Athletic trainers are able to pinpoint which athletes are struggling with being adherent and can implement strategic interventions such as incorporating theoretical frameworks including cognitive appraisal model, personal motivational theory, and theory of planned behavior. Goal setting, imagery, and rehabilitation diaries can also be used to help promote adherence rates. Clinicians need to be aware of the positive and negative impacts that can occur due to the athlete-athletic trainer relationship and the rapport that is built. Clinicians can utilize adherence measures in the clinical practice to educate athletes about the correlation between adherences and clinical outcomes and return to play. Most of the adherence measures are relatively short and easy to administer in the clinical setting. Under and over adherence can cause negative implications for return to play and the athletic trainer can emphasize the importance of following the instructions provided.

Although not evaluated in this study, a question arises if there are other individuals that may affect adherence levels at the D III level as one could also propose that coach's support of injured athletes in sport injury recovery may foster more ideal adherence rates ${ }^{20}$ Coaches that 
enforced consequences for not showing up for treatments improved adherence to sport injury rates. ${ }^{20}$ Further, coaching style can influence adherence rates. ${ }^{20}$ Coaches that had an overbearing coaching style had less adherent athletes compared to coaches with a reasonable and responsible coaching style. ${ }^{20}$ Future research needs to evaluate coach's perspectives on sport injury rehabilitation and the relationships between coaches and athletic trainers at the D III level. Sport injury rehabilitation requires a team of individuals working towards optimal clinical outcomes and a safe return to sport at the highest level possible.

The applicability of adherence literature to the athletic training setting is a concern, thus indicating the need for studies in this setting. ${ }^{18.20}$ This is of key importance to clinicians because of the differences between physical therapy clinics and athletic training rooms. ${ }^{18,20,27}$ Some of these differences include working with athletes versus non-athletes, accessibility to facilities, and contact between the patient and clinician. ${ }^{18,20,27}$ Facilities for athletes are more accessible compared to a clinic for a patient being seen at a clinic. ${ }^{18,20,27}$ Athletic trainers also have the luxury of having daily contact with athletes compared to a physical therapist only being able to see patients two to three times a week. ${ }^{18,20,27}$

\section{Limitations}

The first limitation of this study was that the data was collected from one Division III institution. If more athletes were included from various Division III athletic programs within the same conference may have been able to provide a larger, varying sample size. This could have potentially allowed the original methods to be performed and the potential of having statistically significant findings. Another limitation of this study was the sample size. The original purpose, research questions, and experimental hypotheses had to be altered at the conclusion of data collection. The sample did not yield enough females or individual sport team athletes in order to 
run the original statistical analysis. A larger sample size would have allowed more comparisons to be made. The majority of the sample was football athletes; having a more diversified sport representation of collision, contact, and non-contact sports might have yielded different results. Another limitation included was the testing period. Originally three data points collected on a biweekly basis over the course of a month. Due to time restrictions, only two data points were collected over the course of three weeks. Continuing, the definition of a severe injury may have potentially not allowed athletes to participate in the study. Future research should focus on varying the definition of a sport injury in order to allow more athletes to participate. Finally, there was a level of bias that was experienced during the study. First, there was a selection bias by the athletic trainers selecting athletes that would be compliant with study procedures. In addition there was a level of bias on both the athletic trainer assessing the athlete and the athletes assessing personal behavior. In the future research should focus on blinding the assessor. CONCLUSIONS

Division III athletes at one institution were not over adherent, despite having a high athletic identity. This is a concept that has not been addressed frequently in the literature at the Division III level. In addition, athletic trainers reported moderate to high level of adherence from athletes, which is contrary to reports in the literature. More research needs to be conducted in the traditional clinical athletic training setting at various levels using the athlete and athletic trainer in order to gain more knowledge regarding adherence specifically to the competitive D III athletic population. 


\section{REFERENCES}

1. Powell J, Dompier T. Analysis of injury rates and treatment patterns for time-loss and non-time-loss injuries among collegiate student-athletes. J Athl Train. 2004; 39(1):56-70.

2. Yang J, Tibbetts A, Covassin T, Cheng G, Nayar S, Heiden E. Epidemiology of overuse and acute injuries among competitive collegiate athletes. J Athl Train. 2012;47(2):198-204.

3. Roos K, Marshall S. Definition and usage of the term 'overuse injury' in the US high school and collegiate sport epidemiology literature: A systematic review. Sports Med. 2014;44(3):405-421.

4. Hootman J, Dick R, Agel J. Epidemiology of collegiate injuries for 15 sports: Summary and recommendations for injury prevention initiatives. J Athl Train. 2007;42(2):311-319.

5. Levy A, Polman R, Clough P, McNaughton L. Adherence to sport injury rehabilitation programmes: A conceptual review. Sports Med. 2006;14(2):149-162.

6. Jeansonne J, Hoenig J, Hollander D. Applying sport science theories to increase rehabilitation adherence. Athl Ther Today. 2008;13(3):18-19.

7. Armitage C, Conner M. Social cognition models and health behaviour: A structured review. Psych Health. 2000;15:173-189.

8. Taylor A, May S. Threat and coping appraisal as determinants of compliance with sports injury rehabilitation: An application of protection motivation theory. Sports Sci. 1996; 14: 471-482.

9. Grindley E, Zizzi S, Nasypany A. Use of protection motivation theory, affect, and barriers to understand and predict adherence to outpatient rehabilitation. Phys Ther. 2008;88(12):15291540.

10. Brewer B, Cornelius A, Raalte J, Petitpas A, Sklar J, Pohlman M, Krushell R, Ditmar T. Protection motivation theory and adherence to sport injury rehabilitation revisited. Sport Psych. 2003;17(1):95.

11. Duda J, Smart A, Tappe M. Predictors of adherence in the rehabilitation of athletic injuries: An application of personal investment theory. Sport Exerc Psych. 1989;11:367-381.

12. Cano L, Zafra A, Toro E, and Ros F. Mood states and adherence to rehabilitation for injured athletes. Apunts Med Esport. 2009; 161: 29-37.

13. Podlog L, Gao Z Kenow L, Kleinert J, Granquist M, Newton M, Hannon J. Injury rehabilitation overadherence: Preliminary scale validation and relationships with athletic identity and self-presentation concerns. J Athl Train. 2013;48(3):372-381. 
14. Udry E. Coping and social support among injured athletes following surgery. J Sport Exerc Psych. 1997;19(1):71.

15. Lippke S, Ziegelmann J, Schwarzer R. Behavior intentions and action plans promote physical exercise: A longitudinal study with orthopaedic rehabilitation patients. J Sport Exerc Psych. 2004;26:470-483.

16. Ajzen I. Theory of planned behavior. Organ Behave Human Decision Proc. 1991;50:179211.

17. Meichenbaum D, Turk DC. Facilitating treatment adherence: A practitioner's guidebook. New York: Plenum; 1987:20.

18. Granquist M, Gill D, Appaneal R. Development of a measure of rehabilitation adherence for athletic training. J Sport Rehabil. 2010;19(3):249-267.

19. Fisher A, Mullins S, Frye P. Athletic trainers' attitudes and judgments of injured athletes' rehabilitation adherence. J Athl Train. 1993:28;43-47.

20. Granquist M, Podlog L, Engel J, Newland A. Certified athletic trainers' perspectives on rehabilitation adherence in collegiate athletic training settings. $J$ Sport Rehabil. 2014;23(2):123-133.

21. Brewer B, Avondoglio J, Cornelius A, Raalte J, Brickner J, Petitpas A, Kolt G, Pizzari T, Schoo A, Emery K, and Hatten S. Construct validity and interrater agreement of the sport injury rehabilitation adherence scale. J Sport Rehabil. 2002;11(3):170-178.

22. Levy A, Polman R, Clough P. Adherence to sport injury rehabilitation programs: an integrated psycho-social approach. Sports. 2008;18(6):798-809

23. Bassett S, Phty D. The assessment of patient adherence to physiotherapy rehabilitation. $J$ Physio. 2003;31(2): 60-66.

24. Levy A, Polman R, Clough P, Marchant D, Earie K. Mental toughness as a determinant of beliefs, pain, and adherence in sport injury rehabilitation. J Sport Rehabil. 2006;15(3):246254.

25. Brewer B, Daly J, Raalte V, Petitpas A. A psychometric evaluation of the rehabilitation adherence questionnaire. Sport Exer Psych. 1999;21(2):167.

26. Brewer B. Adherence to sport injury rehabilitation programs. J Applied Sport Psych. 1998;10(1):70-82.

27. Niven A. Rehabilitation adherence in sport injury: sport physiotherapists' perceptions. J Sport Rehabil. 2007;16(2):93-110. 
28. Young K, White P, McTeer W. Body talk: Male athletes reflect on sport, injury, and pain. Socio Sport J. 1994;11(2):175-194.

29. Nixon II H. Explaining pain and injury attitudes and experiences in sport in terms of gender, race, and sport status factors. J Sport Social Issues. 1996;20(1):33-44.

30. Weinberg R, Vernau D, Horn T. Playing through pain and injury: Psychosocial Considerations. J Clin Sport Psych. 2013; 7:41-59.

31. Raudenbush B, Canter R, Corley N, Grayhem R, Koon J, Lilley S, Meyer B, Wilson I. Pain threshold and tolerance differences among intercollegiate athletes: implication of past sports injuries and willingness to compete among sports teams. North Amer J Psych. 2012;14(1):85-94.

32. Eys M, Evans M, Ohlert J, Wolf S, Martin L, Van Bussel M. Cohesion and performance for female and male sport teams. Sport Psych. 2015;29(2):97-109.

33. Visek A, Hurst J, Maxwell J, Watson J. A cross-cultural psychometric evaluation of the athletic identity measurement scale. J Applied Sport Psych. 2008;20(4):473-480.

34. Lamont-Mills A, Christensen S. Athletic identity and its relationship to sport participation levels. J Sci Med Sport. 2006; 9:472-478. 


\section{APPENDICES}




\section{APPENDIX A}

\section{THE PROBLEM}

\section{Research Questions}

Athletic injuries occur in practice and games in National Collegiate Athletic Association (NCAA) sanctioned sports across all three divisions year round. Since the creation of the NCAA, overall, there has been an increase in program participation across all divisions. ${ }^{1,4,35-44}$ This increase in programs includes an increase in the number of athletes participating and therefore increases the incidence of injuries sustained during athletic competition. ${ }^{1,4,35-44}$ The incidence of injury was classified into five general body parts including head/neck, upper extremity, trunk/back, lower extremity, and other/system. ${ }^{1,4,35-44}$ Injuries that were sustained in practice and games were sport dependent. ${ }^{1,4,35-44}$ Due to the injuries that are occurring, practice and games will be missed. During this temporary disqualification from sport, athletes will be actively engaging in rehabilitation programs. The rate in which an athlete is under adherent, adherent, or over adherent can affect treatment goals and ultimately return to play progressions. Therefore, understanding and measuring adherence to the rehabilitation protocol is necessary.

There are various theoretical frameworks that have addressed adherence to rehabilitation programs to advance the knowledge of adherence. ${ }^{5}$ These frameworks include the protection motivational theory ${ }^{5-10}$, personal investment theory, ${ }^{5,6,11}$ attribution theory, ${ }^{5}$ cognitive appraisal models, ${ }^{45,12-14}$ health action process approach, ${ }^{5,7,15}$ and theory of planned behavior. ${ }^{5,7,16}$

The first theoretical model is the attribution theory..$^{5}$ This theory has five major facets; internal, stability, controllability, intentionality, and globality which are thought to determine an athlete's likelihood to adhere to the rehabilitation program. ${ }^{5}$ Patients who believe that their recovery is controllable and stable demonstrate better adherence rates to injury rehabilitation. ${ }^{5}$ 
However, two studies utilizing attribution theory had methodological flaws. ${ }^{5}$ Therefore, research design and measurements need to experience vast improvements in order for attribution theory to be utilized regarding interventions to the rehabilitation program. ${ }^{5}$

Another theory is the theory of planned behavior (TPB). ${ }^{5,7,16} \mathrm{It}$ is an extension of reasoned action. ${ }^{5}$ This theory is mostly used with individuals suffering from low back pain and spinal cord injuries. ${ }^{5,16}$ It has also been applied to job searches, election participation, losing weight, shop lifting, and exercise. ${ }^{16}$ Intention is the primary factor to determine an athlete's ability to engage in behavior change. ${ }^{5,7,16}$ The three major categories of intentions include attitudes toward behavior, subjective norms, and perceived behavioral control. ${ }^{5,716}$ The cognitive appraisal models evaluate stress and coping. ${ }^{5}$ The best developed model noted in the literature is the model presented by Wiese-Bjornstal, Smith, Shaffer, et al. $(1998)^{13}$ to evaluate post-injury behavior. It is an extension of Anderson and Williams $(1988)^{13}$, which evaluates pre-injury behavior. The integrated model includes personal and situational factors on the affect of cognitive and emotional responses to injury. ${ }^{5}$ This theory has been applied to recreational and competitive athletes undergoing knee surgery. ${ }^{5,14}$ This study provided some support of the use of the integrated model in regards to injury rehabilitation. ${ }^{5,14}$ However, a limitation to this model is that it does not address interventions needed at different stages of the rehabilitation process. ${ }^{5,14}$

Health action process approach (HAPA) evaluates three major components; adoption, initiation, and maintenance. ${ }^{5,7,15}$ There are two main phases in this theory. They are the volitional and motivational phase. ${ }^{5,715}$ HAPA has been observed in the development and implementation of an exercise regimen with orthopedic rehabilitation patients along with prevention nutrition, and breast self examination. ${ }^{5,15}$ Limits to research utilizing this theory is non-experimental nature used and involvement of self reporting techniques. ${ }^{15}$ 
Another theoretical model that has been discussed is the protection motivation theory (PMT) ${ }^{5-10}$ In this theory, the athlete is motivated to self-protection from the potential of reinjury and potential negative health set back through the lack of rehabilitation completion. ${ }^{5-10}$ This theory has four major components, which include severity of the health threat, the rate at which the threat can occur, the efficacy of the coping response, and self-efficacy expectancy. ${ }^{5-10}$ PMT has been applied to student athletes in 1996 utilizing the Sports Injury Rehabilitation Belief Survey (SIRBS). ${ }^{5,8}$ The study found some support for the utilization of PMT in predicted adherence behavior. ${ }^{5,8}$ Limitations included heterogenous patient population and rehabilitation protocols. ${ }^{5,8}$ Another study conducted by Brewer ${ }^{5,8}$ evaluated the implementation of PMT in patients recovering from anterior cruciate ligament reconstruction. This study found that coping appraisals showed greater adherence levels compared to threat appraisals. ${ }^{5,10}$

Finally, personal investment theory (PIT) is comprised of three main concepts that determine motivation in an injured athlete. ${ }^{5,6,11}$ These three concepts include personal incentives, sense of self-belief, and perceived options. ${ }^{5,6,11}$ The PIT has been applied to study adherence in intercollegiate athletes. ${ }^{5,11}$ The results from the study support the three main tenets to predict adherence activities. ${ }^{5,11}$ Another study verified the use of PMT with future exercise participation, life satisfaction, and exercise involvement. ${ }^{11}$ Implications from this study included a task-oriented environment, ensure social support, personalization of rehabilitation programs, and maintain high levels of self motivation. ${ }^{4,11}$

As theoretical frameworks continue to evolve so has the research on adherence to rehabilitation programs. Before theoretical frameworks were developed, most of the research conducted had been atheoretical. ${ }^{5}$ These frameworks have helped advanced the knowledge regarding adherence to sports rehabilitation. ${ }^{5}$ Having theoretical frameworks allow for 
educational enhancement along with variables to be studied, research questions, and hypotheses to be formulated in order to create sound studies. ${ }^{5-16}$

The three main theories that have been utilized from the theoretical framework for injury rehabilitation are the personal investment theory (PIT), ${ }^{45,6,11}$ protection motivation theory (PMT), ${ }^{5-10}$ and cognitive appraisal models. ${ }^{5,21-14}$ These theories help evaluate factors that affect adherence levels to the rehabilitation protocol. ${ }^{5-14}$ Studies conducted in sports medicine clinic and home based exercise programs have revealed less than ideal adherence rates. ${ }^{5,8,24,26,27}$ Thus, there is a need for studies in order to improve adherence levels across team and individual sport athletes in order for a safe return to plays and decreased levels of negative health set backs.

In the literature a clear, consistent definition of adherence is lacking. ${ }^{18}$ However, many literature sources use a common definition. That is, "an active, voluntary collaborative involvement of the patient in a mutually acceptable course of behavior to produce a desired preventative or therapeutic result." ${ }^{\text {17 (page 20) }}$ Unfortunately, there is no standardized instrument to measure adherence as there is not a gold standard to measure adherence for a rehabilitation protocol. ${ }^{18}$ Therefore, several instruments have been developed to measure adherence and include the Sport Injury Rehabilitation Adherence Scale (SIRAS), ${ }^{18,21-23}$ Rehabilitation Adherence Questionnaire (RAQ), ${ }^{25}$ Rehabilitation Over Adherence Questionnaire (ROAQ), ${ }^{13}$ the Rehabilitation Adherence Measure for Athletic Training (RAdMAT), ${ }^{18}$ and the athletic identity measurement scale (AIMS). ${ }^{13,33,34}$ These measures use different variations of the Likert scale. ${ }^{13,18,21-23,25,33,34}$ All are measures that are completed by the athlete except the SIRAS and RAdMAT. ${ }^{13,33,34}$ The athletic trainer working with the athlete fills out the survey regarding adherence to the rehabilitation process. ${ }^{18,21-23}$ Of all variables included for adherence, the literature has discussed the length of the rehabilitation as this can affect adherence levels. ${ }^{23}$ The 
main finding is that the longer the rehabilitation the more likely there will be a lack of adherence to the rehabilitation program. ${ }^{23}$ This may be related to longer rehabilitations usually being indicative of a more severe injury. Another reason may be due to the fact that patients find it difficult to dedicate the time to make exercises a part of the daily routine. ${ }^{23}$ Unfortunately, most of the literature examined home based exercise programs or clinic based programs, and not an athletic population. ${ }^{18}$ In addition, there is little information in the literature evaluating a team sport athlete's rehabilitation adherence levels compared to individual sport athlete's level of adherence to the rehabilitation program. It has been noted that males participating in team sports are more likely to perform risky behaviors, such as playing with an injury, in order to

demonstrate masculinity. ${ }^{28,45,46}$ Further, there has been a lack of studies conducted in the athletic training room evaluating adherence levels to rehabilitation. Most studies have focused on under adherence and have not placed a focus on over adherence. In Division III sports, athletes may be more likely to adhere due to the fact that they are playing for the love of sport, and not scholarship benefits. Therefore, based on the information presented, the original research questions raised:

Research Questions

1. Is there a difference in individual sport athletes and team sport athlete's adherence levels?

2. Is there a difference between gender and adherence levels?

However, due to the lack of variation in the participants the following research questions raised:

1. Is there a difference in pre-test and post-test adherence scores?

2. Is there a correlation between athletic identity and over adherent behavior? Experimental Hypotheses

The original experimental hypotheses are: 
1. Athletes who participate in an individual sport will be more likely to over adhere to the rehabilitation process resulting in a longer return to sport timeline.

2. Athletes who participate in team sports are more likely to adhere to the rehabilitation process.

3. Female individual sport athletes are more likely to adhere to the rehabilitation process compared to female team sport athletes.

4. Male individual sport athletes are more likely to adhere to the rehabilitation process compared to male team sport athletes.

5. Overall, women are more likely to adhere to the rehabilitation process compared to males.

However, due to the lack of variation in the patient population, the new experimental hypotheses are as follows:

1. There will be an improvement in adherence behavior from the pre-test to the post-test across all athletic trainer adherence measures.

2. There will be a decrease in over adherent behavior from pre-test to the post-test across the athlete adherence measure.

3. There will be a positive correlation between athletic identity and athletes over adherent behavior.

\section{Assumptions}

1. Participants will answer all surveys honestly and to the best of their ability.

2. The questionnaires being used are valid and reliable.

3. Participants will return questionnaires completed in entirety.

4. Participants will have knowledge regarding injury rehabilitation.

\section{Delimitations}

1. This study utilized injured athletes from one Division III institution and therefore cannot be generalized to other athletic populations. 
2. The results of this study cannot be applied to athletes outside of the Division III population.

Operational Definitions

1. Adherence- "Behaviors an athlete demonstrates by pursuing a course of action that coincides with the recommendations of the athletic trainer. ${ }^{17,18}$

2. Attribution Theory- A theory that focuses on five factors, internal, stability, controllability, intentionality, and globality, which is thought to determine the level of a patients adherence to the rehabilitation program. ${ }^{5}$

3. Cognitive Appraisal Models- Evaluates personal and situational factors, which ultimately affects how an athlete cognitively appraises injury based on what the injury means to the athlete and how severe the athlete perceives the injury to be. ${ }^{5,12-14}$

4. Health Action Process Approach (HAPA)- Focuses on different types of self-efficacy with patient outcomes and threat appraisals. These factors can determine if a patient will initiate and maintain the rehabilitation program. ${ }^{5,7.15}$

5. Individual Sport- A sport that involves one person participating in competition.

6. Non-Time Loss Injury- When an evaluation completed by the certified athletic trainer results in the athlete's concern being defined as a problem, but no sport restrictions are identified. ${ }^{1,2}$

7. Over Adherence- "Behaviors and underlying beliefs of athletes who engage in rehabilitation efforts that exceed practitioner-recommended guidelines." 18

8. Personal Investment Theory (PIT)- Three major facets, personal incentives, sense of self, perceived options, which affect the motivation toward a behavior. In this case, the rehabilitation program. ${ }^{5,6,11}$

9. Protection Motivation Theory (PMT)- An extension of the health belief model. Injured individuals will protect themselves from further harm depending on the perceived severity, beliefs in efficacy of treatment, and the individual's ability to perform exercises to overcome the health aliment. ${ }^{5-10}$

10. Team Sport-A sport that involves more than one athlete to compete in sport competition.

11. Theory of Planned Behavior (TPB)- This theory studies an individual's intention and maintenance to engage in a health related behavior. ${ }^{5,16}$

12. Time Loss Injury- When an evaluation performed by a certified athletic trainer requires an athlete to be withheld from sport. ${ }^{1,2}$ 
13. Under Adherence- Behaviors and underlying beliefs of athletes who do not engage in rehabilitation efforts as prescribed by the athletic trainer. ${ }^{13,18}$

\section{Limitations}

1. Only using injured athletes from one Division III institution.

2. The participants have a biased opinion of injury rehabilitation.

3. The questions in the questionnaire may be misunderstood.

4. The participants may not be in a controlled setting and may be distracted.

5. The busy environment of the athletic training room may deter the ability of the participants to complete the survey to the best of their ability.

\section{Significance of Study}

Studying factors that facilitate adherence to the rehabilitation program is significant because care that is provided to athletes in the athletic training room can improve. The improvement in care will stem from the understanding of how athletes adhere to the rehabilitation process depending on the length of rehabilitation and if the athlete is a team sport athlete or complete individually. This can help the athletic trainer try to implement new therapeutic exercises that are sport specific or modalities in order to keep the rehabilitation process from becoming mundane to the athlete, thus improving return to sport rates in a safe and effective manner.

Besides the benefits that can be provided to the athletes, the athletic trainer and coaching staff will benefit from this study as well. Athletic trainers can develop new exercises that are sport specific or mimic sport movements in order to keep athletes who have a long rehabilitation program adherent to the program. Athletic trainers can also take time to educate the athletes on the healing process, the individual injury, and the rehabilitation process. For individuals who have a lengthy rehabilitation, this knowledge may help maintain a more appropriate level of 
adherence. In addition, coaches will benefit from this study as well. Coaches want the athletes to return to sport as quickly as possible. They can play an important role in encouraging the athletes to adhere to the rehabilitation program.

Athletic trainer, athlete, and coach education components will vary in content. Athletic trainers education component will include information regarding adherence to the rehabilitation program, how to identify individuals who are under or over adhering, and education in order to prevent set backs or re-injury during the rehabilitation process. Athletic trainers can receive information through conferences, seminars, publications, and presentations. Athlete education will consist of a handout, which highlights the healing and rehabilitation processes. This will highlight warning signs of potential set backs in order to prevent re-injury. The goal of education will be for patient awareness of physiological processes occurring in the body preventing return to sport or living activities. In addition, some interventions to help promote adherence will be described in order for patients to maintain positive rehabilitation experience. Coaching education components will also be in the form of a flyer. It will explain the importance of proper athlete adherence to the rehabilitation process and ways they can help support or promote athlete adherence to rehabilitation protocol prescribed to by athletic trainer. 


\section{APPENDIX B \\ REVIEW OF LITERATURE}

Introduction

Having athletes safely return to sport is an athletic trainers primary goal while working with National Collegiate Athletic Association (NCAA) athletic teams. Due to the unpredictable nature of sports, injuries are bound to occur. These injuries may temporarily withhold athletes from sport and result in the athlete actively participating in a rehabilitation program. Depending on the compliance level of the athlete to the rehabilitation progress can affect treatment outcomes and ultimately return to play. Therefore, the purpose of this literature review is to discuss the literature regarding epidemiology of injuries, theoretical frameworks of rehabilitation adherence, measurement tools to assess adherence, and athletic trainers perceptions of adherence to rehabilitation.

Epidemiology of Injuries

The NCAA has collected descriptive epidemiology data utilizing the injury surveillance system from 1988-1989 through 2002-2003, and is currently working on epidemiology studies

for 2014-2015. 4,35-44 These studies collected data regarding games and practice athlete exposure, injury rate by activity, division, and season, body parts injured most often and specific injuries, mechanism of injury, severe injuries: 10+ days of activity time loss, and game injuries. ${ }^{4,35-44}$ Sports that the NCAA collected data for include women's soccer, women's lacrosse, women's basketball, women's volleyball, women's softball, men's wrestling, men's soccer, men's basketball, men's football, and men's baseball.,35-44

Through the surveillance system from 1988-1989 through 2002-2003 it has been demonstrated that every NCAA sport excluding wrestling has experienced an increase in 
participation. ${ }^{35-44}$ Table B1 exemplifies the number of games, athletes whom participate in games, practices, and athletes whom participate in practice in NCAA sports at the DIII level. ${ }^{35-44}$ The sports that reported the highest number of games are baseball, softball, and volleyball. ${ }^{35-44}$ The sports that reported the lowest number of games include football, men's soccer, and women's soccer. ${ }^{35-44}$ The sports that had the highest number of athletes participate in a game included football, women's lacrosse, women's soccer, and men's soccer. ${ }^{35-44}$ The sports that reported the lowest number of athletes to participate in a game include wrestling, women's volleyball, and women's basketball. ${ }^{35-44}$ The sports that reported the highest number of practices include men's basketball, wrestling, and football. ${ }^{35-44}$ The sports that reported the lowest number of practices included women's volleyball, women's soccer, and softball. ${ }^{35-44}$ The sports that reported the most athletes participating in practice included football, baseball, and men's soccer. ${ }^{35-44}$ The sports that reported the least amount of athletes participating per practice included women's volleyball, women's basketball, softball, and men's basketball. ${ }^{35-44}$ $\underline{\text { Table B1.DIII Games/Athletes Per Game/Practices/Athletes Per Practice for NCAA Sports }}$

$\begin{array}{llll}\text { Sport Games } & \text { Athletes Per Game Practices } & \text { Athletes Per }\end{array}$

$\begin{array}{ccccc}\text { Women's Soccer } & 17 & 16 & 44 & \text { Practice } \\ \text { Men's Soccer } & 17 & 16 & 48 & 20 \\ \text { Women's } & 24 & 10 & 65 & 13 \\ \text { Basketball } & & 11 & 73 & 16 \\ \text { Men's Basketball } & 24 & 12 & 47 & 16 \\ \text { Softball } & 32 & 13 & 52 & 26 \\ \text { Baseball } & 34 & 16 & 48 & 20 \\ \text { Women's Lacrosse } & 14 & 9 & 44 & 13 \\ \text { Women's } & 30 & 48 & 68 & 78 \\ \text { Volleyball } & 9 & 9 & 72 & 17 \\ \text { Football } & 19 & & & \\ \text { Wrestling } & & & \end{array}$


It is consistently reported through the NCAA surveillance system that injury rates were higher in the preseason compared to in season, and that in season injury rates were higher compared to most season injury rates. ${ }^{35-44}$ Athletes were more likely to sustain an injury during a game compared to practice. ${ }^{35-44}$ This may be due to the fact that athletes did not follow conditioning packets, and coming into the pre-season unprepared for the demands of sport. ${ }^{35-44}$ Games are more competitive in nature compared to practices potentially explaining the increase of injury compared to practice. ${ }^{35-44}$ Table B2 displays pre-season games and practices with associated injury rates. ${ }^{35-44}$ The sports that reported the highest total number of games for the pre-season included women's volleyball, softball, and baseball. ${ }^{35-44}$ The teams that reported the lowest total number of games included wrestling, women's lacrosse, and women's soccer. ${ }^{35-44}$ The sports that reported the highest game injury rate per 1000AE include women's soccer, wrestling, and men's soccer. ${ }^{35-44}$ While the lowest game injury rate per 1000AE included women's volleyball, softball, and baseball. ${ }^{35-44}$ The highest total number of practices reported included baseball, men's basketball, and softball. ${ }^{35-44}$ The sports that reported the lowest include wrestling, women's soccer, and women's volleyball. ${ }^{35-44}$ Highest practice injury rates per 1000AE included women's soccer, men's soccer, and women's volleyball. ${ }^{35-44}$ Lowest injury rates included baseball, softball, and women's lacrosse. ${ }^{35-44}$

Table B2. DIII Preseason Games and Practices with Associated Injury Rates by NCAA

$\begin{array}{ccccc}\text { Sport } & \begin{array}{c}\text { Total No. of } \\ \text { Games } \\ \text { Reported }\end{array} & \begin{array}{c}\text { Game Injury } \\ \text { Rate per } \\ 1000 \mathrm{AE}\end{array} & \begin{array}{c}\text { Total No. of } \\ \text { Practices } \\ \text { Reported }\end{array} & \begin{array}{c}\text { Practice Injury } \\ \text { Rate per } \\ 1000 \mathrm{AE}\end{array} \\ \begin{array}{c}\text { Women's } \\ 263\end{array} & 24.04 & 7,495 & 9.1 \\ \begin{array}{c}\text { Soccer } \\ \text { Men's Soccer }\end{array} & 353 & 15.21 & 8,951 & 7.76 \\ \text { Women's } & 371 & 6.49 & 13,828 & 5.60 \\ \text { Basketball } & & & & \\ \text { Men's } & 422 & 8.3 & 14,773 & 6.6 \\ \text { Basketball } & & & & \end{array}$




$\begin{array}{ccccc}\text { Softball } & 847 & 2.57 & 14,296 & 2.94 \\ \text { Baseball } & 799 & 3.38 & 15,922 & 2.15 \\ \text { Women's } & 172 & 6.28 & 8,939 & 3.53 \\ \text { Lacrosse } & & & & \\ \text { Women's } & 885 & 2.28 & 8,666 & 5.79 \\ \text { Volleyball } & & & & \\ \text { Football } & 281 & 9.14 & 13,872 & 7.85 \\ \text { Wrestling } & 113 & 18.5 & 5081 & 7.3\end{array}$

Table B3 displays in-season games and practices with associated injury rates at the DIII level. ${ }^{35-44}$ Similar to the pre-season, baseball and women's volleyball reported the highest total number of games reported. ${ }^{35-44}$ In addition, wrestling and women's lacrosse reported the lowest total number of games reported. ${ }^{35-44}$ The game injury rate per 1000AE for the pre-season and inseason are similar as wrestling and men's soccer reported the highest rates along with football. ${ }^{35-}$ 44 The lowest game injury rate for the pre-season and in-season were identical with women's volleyball, softball, and baseball being represented. ${ }^{35-44}$ The sports that reported the highest total number of practices for the in-season included men's basketball, women's basketball, and football. ${ }^{35-44}$ The lowest number of practices reported was for women's lacrosse, softball, and wrestling. ${ }^{35-44}$ The sports that represented the highest practice injury rate per 1000AE include wrestling, women's soccer, and women's volleyball. ${ }^{35-44}$ Sports that reported the lowest practice injury rates for the in-season were identical to the pre-season sports, which are baseball, softball, and women's lacrosse. ${ }^{35-44}$

Table B3. DIII In Season Games and Practices with Associated Injury Rates by NCAA

$\begin{array}{ccccc}\text { Sport } & \begin{array}{c}\text { Total No. of } \\ \text { Games } \\ \text { Reported }\end{array} & \begin{array}{c}\text { Game Injury } \\ \text { Rate per } \\ \text { 1000AE }\end{array} & \begin{array}{c}\text { Total No. of } \\ \text { Practices } \\ \text { Reported }\end{array} & \begin{array}{c}\text { Practice Injury } \\ \text { Rate per } \\ \text { 1000AE }\end{array} \\ \text { Women's } & 8,464 & 15.45 & 14,636 & 2.88 \\ \text { Soccer } & & 15.83 & 17,589 & 2.26 \\ \text { Men's Soccer } & 9,429 & 6.67 & 29,309 & 2.48 \\ \text { Women's } & 15,245 & & & \\ \text { Basketball } & & & \end{array}$




\begin{tabular}{ccccc} 
Men's & 14,523 & 9.1 & 30,376 & 2.8 \\
Basketball & & & & \\
Softball & 14,556 & 4.35 & 11,083 & 1.53 \\
Baseball & 17,191 & 5.10 & 13,988 & 0.95 \\
Women's & 4,273 & 6.22 & 7,454 & 2.19 \\
Lacrosse & & & & \\
Women's & 15,784 & 4.00 & 17,608 & 2.60 \\
Volleyball & & & & \\
Football & 5,602 & 34.31 & 28,377 & 2.32 \\
Wrestling & 4,280 & 21.7 & 13,425 & 4.4 \\
\hline
\end{tabular}

Table B4 exemplifies the DIII post-season games and practices with associated injury rates by NCAA. ${ }^{35-44}$ The sports that reported the highest total number of games included baseball, softball, and women's lacrosse. ${ }^{35-44}$ This is similar to the sports represented during the competitive season. ${ }^{35-44}$ The sports that reported the lowest number of games were identical for the in-season and post-season. ${ }^{35-44}$ These sports included football, women's lacrosse, and wrestling. ${ }^{35-44}$ The highest and lowest reported game injury rate per 1000AE was identical for in season and post season. ${ }^{35-44}$ The sports that represented the highest included football, wrestling, and men's soccer. ${ }^{35-44}$ The sports that were represented the lowest included softball, women's volleyball, and baseball. ${ }^{35-44}$ The sports that reported the highest total number of practices reported included men's basketball, wrestling, and women's volleyball. ${ }^{35-44}$ The sports that reported the lowest total number of practices included women's lacrosse, football, and softball. ${ }^{35-}$ ${ }^{44}$ The highest practice injury rate per 1000AE were men's basketball, wrestling, and women's basketball. ${ }^{35-44}$ The sports with the lowest reported practice injury rates include softball, women's lacrosse, and football. ${ }^{35-44}$

Table B4. DIII Post Season Games and Practices with Associated Injury Rates by NCAA

\begin{tabular}{|c|c|c|c|c|}
\hline Sport & $\begin{array}{l}\text { Total No. of } \\
\text { Games } \\
\text { Reported }\end{array}$ & $\begin{array}{l}\text { Game Injury } \\
\text { Rate per } \\
1000 \mathrm{AE}\end{array}$ & $\begin{array}{c}\text { Total No. of } \\
\text { Practices } \\
\text { Reported }\end{array}$ & $\begin{array}{l}\text { Practice Injury } \\
\text { Rate per } \\
\text { 1000AE }\end{array}$ \\
\hline
\end{tabular}




\begin{tabular}{ccccc} 
Women's & 399 & 10.41 & 741 & 1.01 \\
$\begin{array}{c}\text { Soccer } \\
\text { Men's Soccer }\end{array}$ & 386 & 12.84 & 849 & 0.83 \\
Women's & 655 & 5.26 & 1,113 & 1.12 \\
$\begin{array}{c}\text { Basketball } \\
\text { Men's }\end{array}$ & 760 & 7.7 & 1,483 & 2.1 \\
Basketball & & & & \\
Softball & 855 & 2.11 & 680 & 0.47 \\
Baseball & 907 & 2.92 & 813 & 0.83 \\
Women's & 300 & 3.77 & 559 & 0.64 \\
Lacrosse & & & & \\
Women's & 796 & 2.57 & 1,189 & 1.00 \\
Volleyball & & & & \\
Football & 137 & 24.44 & 691 & 0.75 \\
Wrestling & 331 & 18.3 & 1,391 & 1.9 \\
\hline
\end{tabular}

Table B5 summarizes the total number of games and practices with associated injury rates by the NCAA at the DIII level. ${ }^{35-44}$ In total, the sports that reported the highest total number of games included women's volleyball, baseball, and softball. ${ }^{35-44}$ The sports that reported the lowest included wrestling, women's lacrosse, and football. ${ }^{35-44}$ The sports that reported the highest game injury rate per $10000 \mathrm{AE}$ were football, wrestling, and men's soccer. ${ }^{35-}$ ${ }^{44}$ Women's volleyball, softball, and baseball. Reported the lowest ${ }^{35-44}$ The highest total number of practices reported included men's basketball, women's basketball, and football. ${ }^{35-44}$ The sports that reported the lowest included women's lacrosse, wrestling, and women's soccer. ${ }^{35-44}$ Practice injury rates per 1000AE were the highest for women's soccer, wrestling, and football. ${ }^{35-}$ ${ }^{44}$ The sports that reported the lowest included baseball, softball, and women's lacrosse. ${ }^{35-44}$ Table B5. DIII Total Games and Practices with Associated Injury Rates by NCAA

\begin{tabular}{|c|c|c|c|c|}
\hline Sport & $\begin{array}{l}\text { Total No. of } \\
\text { Games }\end{array}$ & $\begin{array}{l}\text { Game Injury } \\
\text { Rate per }\end{array}$ & $\begin{array}{l}\text { Total No. of } \\
\text { Practices }\end{array}$ & $\begin{array}{l}\text { Practice Injury } \\
\text { Rate per }\end{array}$ \\
\hline & Reported & $1000 \mathrm{AE}$ & Reported & $1000 \mathrm{AE}$ \\
\hline $\begin{array}{l}\text { Women's } \\
\text { Soccer }\end{array}$ & 9,129 & 15.21 & 22,801 & 5.25 \\
\hline Men's Soccer & 10,168 & 15.76 & 27,389 & 4.15 \\
\hline
\end{tabular}




\begin{tabular}{ccccc}
$\begin{array}{c}\text { Women's } \\
\text { Basketball }\end{array}$ & 16,271 & 6.62 & 44,250 & 3.45 \\
$\begin{array}{c}\text { Men's } \\
\text { Basketball }\end{array}$ & 15,705 & 9.0 & 46,632 & 4.0 \\
Softball & 16,258 & 4.14 & 26,059 & 2.28 \\
Baseball & 18,897 & 4.85 & 30,723 & 1.59 \\
Women's & 4,745 & 6.07 & 16,952 & 2.87 \\
Lacrosse & & & & \\
Women's & 17,476 & 4.01 & 27,463 & 3.70 \\
Volleyball & & & & \\
Football & 6,020 & 32.89 & 42,940 & 4.18 \\
Wrestling & 4,744 & 21.3 & 19,907 & 5.1 \\
\hline
\end{tabular}

Tables B6 and B7 display the percentage of game and practice injuries that occurred to the upper and lower extremity. ${ }^{35-44}$ In a game setting the sports that sustained the most upper extremity injuries included baseball, softball, and wrestling. ${ }^{35-44}$ The sports that sustained the lower percentage of upper extremity injuries in a game setting included women's soccer, men's soccer, and women's lacrosse. ${ }^{35-44}$ In a practice setting the sports that reported the highest number of upper extremity injuries include baseball, softball, wrestling, and football, with the lowest percentage in women's soccer, men's soccer, and women's lacrosse. ${ }^{35-44}$ The sports that reported the highest percentage of game injuries to the lower extremity included women's soccer, men's soccer, and women's lacrosse. ${ }^{35-44}$ The sports that reported the lowest percentages included baseball, wrestling, and softball. ${ }^{35-44}$ The highest percentage of injuries to the lower extremity during practices included women's soccer, men's soccer, and women's basketball. ${ }^{35-44}$ The sports that reported the lowest percentages included wrestling, baseball, and softball. ${ }^{35-44}$ Overall, the report percentages for the lower extremity where much higher compared to the upper extremity. ${ }^{35-44}$ Table B6. Percentage of Game and Practice Injuries Upper Extremity

$\begin{array}{ccc}\text { Sport } & \text { Games } & \text { Practices } \\ \text { Women's Soccer } & 6.3 & 4.2\end{array}$




$\begin{array}{ccc}\text { Men's Soccer } & 6.8 & 5.3 \\ \text { Women's Basketball } & 14.1 & 10.4 \\ \text { Men's Basketball } & 14.1 & 11.1 \\ \text { Softball } & 33.1 & 33.0 \\ \text { Baseball } & 44.6 & 46.4 \\ \text { Women's Lacrosse } & 8.9 & 5.9 \\ \text { Women's Volleyball } & 21.4 & 18.7 \\ \text { Football } & 22.6 & 20.1 \\ \text { Wrestling } & 26.1 & 20.1\end{array}$

Table B7. Percentage of Game and Practice Injuries Lower Extremity

$\begin{array}{ccc}\text { Sport } & \text { Games } & \text { Practices } \\ \text { Women's Soccer } & 67.8 & 72.0 \\ \text { Men's Soccer } & 67.3 & 70.7 \\ \text { Women's Basketball } & 60.8 & 65.6 \\ \text { Men's Basketball } & 57.9 & 60.6 \\ \text { Softball } & 43.3 & 40.8 \\ \text { Baseball } & 35.2 & 31.7 \\ \text { Women's Lacrosse } & 61.0 & 64.3 \\ \text { Women's Volleyball } & 58.7 & 55.9 \\ \text { Football } & 54.7 & 50.8 \\ \text { Wrestling } & 40.3 & 31.3\end{array}$

Finally, Table B8 represents the percent of injuries that required 10 or more days of time loss from sport. ${ }^{35-44}$ The sports that reported the highest percent of injuries requiring time lost from sport for games include wrestling, football, and women's basketball. ${ }^{35-44}$ The lowest percentage of injuries that required time loss included men's basketball, men's soccer, and women's soccer. ${ }^{35-44}$ The sports that reported the highest percent of injuries that required time loss from practice participation included wrestling, women's lacrosse, and football. ${ }^{35-44}$ The lowest percent of injuries that required time loss from practice includes men's soccer, women's soccer, and women's volleyball. ${ }^{35-44}$

Table B8. Percent of Injuries that Required 10+ Days of Time Loss

$\begin{array}{ccc}\text { Sport } & \text { Games } & \text { Practices } \\ \text { Women's Soccer } & 21.8 \% & 16.5 \%\end{array}$




$\begin{array}{ccc}\text { Men's Soccer } & 18.7 \% & 14.6 \% \\ \text { Women's Basketball } & 25.3 \% & 23.6 \% \\ \text { Men's Basketball } & 18.0 \% & 18.0 \% \\ \text { Softball } & 24.8 \% & 22.0 \% \\ \text { Baseball } & 25.2 \% & 25.0 \% \\ \text { Women's Lacrosse } & 21.9 \% & 23.9 \% \\ \text { Women's Volleyball } & 23.0 \% & 19.0 \% \\ \text { Football } & 27.0 \% & 24.9 \% \\ \text { Wrestling } & 34.0 \% & 28.0 \%\end{array}$

Powell ${ }^{1}$ conducted a study evaluating injury rates and treatment rates for time-loss and non-time loss injuries among collegiate athletes. The findings from this study report that 450,000 athletes competed in college sports, furthermore, 137,000 athletes participated at the Division III level. ${ }^{1}$ At the time of study, the National Athletic Training Association (NATA) had 23,000 certified athletic training members, $20 \%$ of those members stated that they were employed by either colleges or universities. ${ }^{1,4}$ Athletic trainers are the primary health care provider for college athletes and are typically present at time of injury. ${ }^{1}$ Athletic trainers in this setting exemplify a ratio of one athletic trainer per 100 athletes. ${ }^{1}$ Due to the increase in participation in collegiate athletics, athlete exposure to injury increases, thus increasing the demands of evaluation, diagnosis, and rehabilitation skills of the athletic training staff. ${ }^{1}$

Results from this study indicated that of the 68,497 injuries that were reported, $79.9 \%$ of them resulted in no time loss from athletic participation. ${ }^{1}$ For males $77.7 \%$ of injuries were nontime loss injuries and $83.6 \%$ of female injuries. ${ }^{1}$ Football reported the highest incidence of nontime loss injuries (52.1\%) and time-loss injuries (57.9\%). ${ }^{1}$ Non-time loss injuries were more prevalent for males than time loss injuries. ${ }^{1}$ Non-time loss injuries for males resulted in $57.9 \%$ of treatments. ${ }^{1}$ The average number of treatments for injury was 10.7 , with an average of 7.2 treatments for non-time loss injury treatment. ${ }^{1}$ The average amount of treatments for time loss injuries were 22.7 treatments. $^{1}$ 
For women's sports, soccer had the highest non-time loss injuries (17.9\%) and time loss injuries $(22 \%) .{ }^{1}$ Like men's sports, women's sports also reported higher rates of non-time loss injuries compared to time loss injuries. ${ }^{1}$ Women's treatment for non-time loss injuries resulted in $66.1 \%$ of treatments performed. ${ }^{1}$ There was an average of 12.7 treatments per injury, with nontime loss injuries requiring an average of 10 treatments and time loss injuries requiring an average of 26.2 treatments. $^{1}$

In the Division III level of participation, women's sports experienced higher rates of nontime loss injuries, however, men's sport programs experienced a higher level of time loss injuries. ${ }^{1}$ For men's sports, wrestling experienced the highest rate of injury for non-time loss injuries followed by basketball, football, and soccer. ${ }^{1}$ The sports that experienced the highest rate of time loss injuries included football, soccer, wrestling, and soccer. ${ }^{1}$ Of all the treatments carried out in the Division III institutions, $55.4 \%$ were performed on males. ${ }^{1}$ The average number of treatments performed per injury was 7.7 treatments. ${ }^{1}$ For non-time loss injuries, 6.1 treatments were performed compared to time loss injuries required 11.8 treatments. ${ }^{1}$

For Division III women's sports the teams that reported the highest non-time loss injuries were volleyball followed by basketball, and soccer. ${ }^{1}$ The sports that reported the highest rate for time loss injuries include soccer followed by basketball. ${ }^{1}$ Of all the treatments that were performed at the Division III level, $44.6 \%$ were conducted on women. ${ }^{1}$ The average number of treatments that were performed per injury was $9.4 .{ }^{1}$ For non-time loss injuries, 7.3 treatments were performed while 18.2 treatments were required for time loss injuries. ${ }^{1}$

This research exemplifies that regardless of time loss or non-time loss injuries, rehabilitation programs are utilized in order to keep athletes healthy enough to continue to participate in sport or to safely return athletes to sport after temporary disqualification. 


\section{Theoretical Frameworks}

Since there are injuries in practices and games that are withholding athletes from sport for at least ten consecutive days there is a need for athletes to actively participate in a rehabilitation program. The athlete's ability to adhere to the rehabilitation program affects the ability to return to play. In order to better understand the concept of adherence, it is important to understand the theoretical frameworks concerning adherence. These theoretical models help enhance the concept of adherence to rehabilitation. ${ }^{5}$ The various frameworks allow for research questions to be formulated, hypothesis to be tested, and variables to be evaluated. ${ }^{5-16}$

One such framework is the personal investment theory. ${ }^{5,6,11}$ This theory was originally created by Maehr and Braskamp in 1986. ${ }^{11}$ Personal investment theory (PIT) has been used to measure adherence rates in collegiate athletes. ${ }^{5,11}$ This theory is comprised of three main concepts that determine motivation in an injured athlete. ${ }^{5,6,11}$ These three factors include personal incentives, sense of self-belief, and perceived options. ${ }^{5,6,11}$ Personal incentives include the athlete's short and long-term goals. ${ }^{5,11}$ This first component of PIT can be divided into four main categories, which include ego incentives, task incentives, social incentives, and extrinsic rewards. ${ }^{11}$ The second factor, sense of self, involves the athlete's ideologies, thoughts, and feelings they hold about him or herself. ${ }^{5,11}$ There are four-sub categories to this main factor, which include perception of competence, self-reliance, goal direction, and self-identity. ${ }^{11}$ Finally, a perceived option is defined as different options that the athlete has during his or her treatment. ${ }^{5,6,11}$ Components of perceived options is that options have to be perceived as available, appropriate, and interest or investment in the behavior. ${ }^{11}$

There have been studies that support the use of PIT in physical activity, expected physical exercise participation, and life satisfaction with middle aged and older adults. ${ }^{11}$ In these 
studies, the three main components were related to adherence levels. ${ }^{11}$ In Duda's ${ }^{11}$ study evaluating PIT in a group of collegiate athletes, it suggested that adherence was related to the athletes belief in treatment, social support, self-motivation, and task involvement. ${ }^{11}$ He noted that athletes who displayed less self-motivation were more likely to exemplify poorer adherence rates. ${ }^{11}$ His study supported the literature by stating that athletic trainers play an integral part in sports rehabilitation adherence by creating an optimal environment and promoting effects of treatment. $^{11}$

Implications of this theory can include promoting a task-orientation and goal setting in the rehabilitation location. ${ }^{5,6,11}$ In addition, in order to promote effective social support, injury groups should be created so that athletes have a safe place to voice concerns and questions. ${ }^{5,11}$ Finally, rehabilitation programs should be individualized to each athlete by the health care professional. $^{5,11}$

Another framework that has been discussed in the literature includes the protection motivation theory (PMT). ${ }^{5-10}$ This theory is an extension of the health belief model. ${ }^{10}$ Individuals desire to protect themselves from a health aliment; however, this is affected by perception of how serious and how likely or unlikely to sustain the aliment. ${ }^{5-10}$ The ability to overcome the health threat is determined by the belief of treatment effectiveness and the ability to engage in activities that will help improve health status. ${ }^{5-10}$ There are two cognitive appraisal processes that occur in this model. They are threat and coping appraisals. ${ }^{7,8}$ Threat appraisals are classified as the patients' perceived severity and susceptibility. ${ }^{7,8}$ Coping appraisals occur when the individual realizes the positive effects of rehabilitation and ability to perform exercises. $^{7,8}$ 
Taylor and May ${ }^{8}$ applied the PMT to sports injury adherence with student athletes in 1996. The investigators utilized the Sports Injury Rehabilitation Belief Survey (SIRBS) to capture the essence of the premise of the theory. ${ }^{5,8}$ The results of this study suggested partial support for PMT, revealing that severity and susceptibility predicted lower levels of adherence. ${ }^{5,8}$ Higher levels of adherence were related to the individuals self efficacy and belief in treatment. ${ }^{5,8}$

Brewer et al. ${ }^{10}$ in 2003 applied concepts from PMT to patients recovering from anterior cruciate ligament reconstruction. The results of the study revealed that coping appraisals compared to threat appraisals demonstrated higher levels of rehabilitation adherence..$^{5,10}$ Comparable to the Taylor and May study, Brewer also found that treatment efficacy lead to higher levels of adherence. . $^{5,10}$

The application of PMT is limited in that it has only been applied to heterogeneous populations. ${ }^{5,8,10}$ The injured athletes rehabilitation beliefs survey (SIRBS) is a measurement tool that assessed the concepts of PMT and the application to clinic based rehabilitation programs. ${ }^{45,8,10}$ In addition, based on results of the studies, it is important that athletic trainers promote the importance of rehabilitation exercises and modalities to the patient, improve the patients belief in the ability to perform exercises, and to highlight the severity and susceptibility to health due to poor adherence to rehabilitation. ${ }^{5,8,10}$

A different theoretic framework of adherence to rehabilitation included the attribution theory. ${ }^{5}$ The premise of this theory is that there are five components that affect an athlete's ability to adhere or not adhere to a rehabilitation protocol. ${ }^{5}$ These factors include internal, stability, controllability, intentionality, and globality. ${ }^{5}$ Patients who believe that rehabilitation is controllable and stable, two of the five main components, tend to adhere better to prescribed rehabilitation programs. ${ }^{5}$ The results from two studies references that in order for athletes to 
respond in a way that is consistent with beliefs, the attributions must be internal, stable, and controllable. ${ }^{5}$ Rehabilitation diaries have been beneficial to help track progress, attainment of goals, and to promote positive thoughts and feelings regarding the rehabilitation process. ${ }^{5}$ However, much work regarding research design and measurements need to be explored to make the attribution theory a sound framework in order to implement interventions to promote adherence for injured athletes. $^{5}$

Another model that has been discussed in the literature includes the cognitive appraisal model by Wiese-Bjornstal et $\mathrm{al}^{5,12-14}$ This model was constructed in 1998, and focused on post injury behavior in the athlete. ${ }^{12-14}$ Primarily, the model is a continuation of Williams and Anderson's pre-injury model constructed in $1988 .{ }^{5}$ Personal and situational factors that will affect how an athlete cognitively appraises injury is the main focus. ${ }^{5,12-14}$ Cognitive appraisals of the injury will depend on the meaning of the injury to the athlete, the consequences of the injury, and the athletes' ability to cope. ${ }^{5,12-14}$ The cognitive appraisals can be classified as either positive or negative. This ultimately affects emotional and behavioral responses. ${ }^{5,12-14}$ If an athlete has a more positive appraisal of injury, adherence to rehabilitation is higher compared to an athlete who had a negative cognitive appraisal of the injury. ${ }^{5,12-14}$ Athlete's who have a negative appraisal of injury typically work through negative thoughts and emotions within a two-week period. ${ }^{5,12-14}$ However, if negative thoughts, emotions, and behaviors are still present after a two week period, referral to a sport psychologist consultant may be beneficial to help the athlete address negative thoughts, emotions, and behavior ${ }^{5,12-14}$ In addition, if an athlete has a high selfpresentation levels, high athletic identity, and personality traits, over adherence may be evident, in order to attempt to return to play faster. ${ }^{5,12-14}$ Athletes' usually engage in these behaviors 
when experiencing approval from teammates, coaches, and fans. However, this model lacks psychological interventions and implementations., ${ }^{5,12}$

A different theoretical framework that has been discussed in the literature is the health action process approach (HAPA). ${ }^{5,715}$ This theory is classified as a multi-staged theory, meaning that it has various stages. ${ }^{7}$ HAPA applies adopting and maintaining physical behavior during rehabilitation. ${ }^{5,7,15}$ This theory has three main tenets, which include adoption, initiation, and maintenance..$^{5,715}$ There are two phases in HAPA. ${ }^{5,715}$ They are the volitional phase and motivational phase..$^{5,715}$ The volitional phase is characterized as a planning stage. ${ }^{5,7,15}$ This stage involves individuals preparing to begin a new activity, situational factors, cognitive appraisal, and behavioral factors. ${ }^{5,715}$ Self-efficacy plays a major role during the volitional phase. ${ }^{5,7,15}$ Intended behaviors must be planned, including when and where, and how. $5,7,15$

Lippke et al. ${ }^{15}$ in 2004 applied the theory of HAPA to orthopedic rehabilitation patients implementing and adopting a physical exercise program. It appeared that self-efficacy and outcome expectancies are directly related to intentions and planning of an exercise program..$^{5,15}$ Implications from this study included promoting self-efficacy in patients during the motivational phase to promote outcome expectancies. ${ }^{5,15}$ HAPA has also been applied to food choice, physical exercise, nutrition, and breast examination..$^{5,15}$

Finally, the last theoretical framework is the theory of planned behavior (TPB). TPB is a continuation of the theory of reasoned behavior. ${ }^{5,7,16}$ This theory has been utilized with patients who are suffering from low back pain and spinal cord injuries. ${ }^{5}$ The focus of TPB is on an individuals intentional planning to complete or not complete rehabilitation exercises. ${ }^{5,7,16}$ The athlete's intentions are affected by attitudes toward the behavior, subjective norms, and perceived behavioral control. ${ }^{5,7,16}$ The stronger intention that one has the more likely they are to 
actually perform the skill. ${ }^{16}$ However, there are some factors such as time, money, skill, and accessibility, which can deter an individual from completing rehabilitation exercises. $5,7,16$ However, intention and ability determine if the behavior is achieved. ${ }^{7,16}$ How the individual perceives ability to perform the action will determine how likely to actually perform the action. ${ }^{16}$ The theory of planned behavior has been applied in activities such as job searches, video games, problem drinking, leisure activities, election participation, losing weight, obtaining an A in a class, shop lifting, and exercise. ${ }^{16}$ However, there are methodological issues regarding the TPB. ${ }^{5}$ These limitations include cross-sectional research, self reports, and multiple regression models used, which inhibits an overall model of best fit to be implemented. ${ }^{5}$ Future research is needed to assess the effectiveness of applying TPB to rehabilitation adherence. ${ }^{5,7,16}$ Measurements of Adherence

In the literature there is no clear definition of adherence. ${ }^{18}$ However, a common definition of adherence, "an active, voluntary collaborative involvement of the patient in a mutually acceptable course of behavior to produce a desired preventative or therapeutic result." ${ }^{17(\text { page20) }}$ There is no gold standard that is utilized to measure adherence to the rehabilitation process. ${ }^{18}$ There is a need to study adherence to the rehabilitation program due to the large range of $40 \%-91 \%$ compliance rates reported in the literature 20,21

The literature relies on different instruments to evaluate adherence by utilizing surveys. 13,18,21-23,25 Commonly utilized tools to measure adherence include Sport Injury Rehabilitation Adherence Scale (SIRAS), ${ }^{5-14,18,21-23}$ Rehabilitation Adherence Questionnaire (RAQ), ${ }^{25}$ Rehabilitation Over Adherence Questionnaire (ROAQ),${ }^{13}$ Rehabilitation Adherence Measure for Athletic Training (RADMAT), ${ }^{18}$ and the Athletic Identity Measurement Scale (AIMS). ${ }^{13,30,33,34}$ 
The 3-item Sport Injury Rehabilitation Adherence Scale (SIRAS) evaluates more than just the patient being present for rehabilitation sessions. ${ }^{18,21-24}$ The scale utilizes a five point Likert scale to evaluate the effort the patient exerts while performing therapeutic exercises, how well the patient follows instructions from the health care provider, and ability to manage constructive criticism regarding the rehabilitation program. ${ }^{18,21-24}$ The instrument is unique in that the health care professional and not the patient completes this instrument. ${ }^{18,21-24}$ This instrument has been utilized to measure adherence in sport-injury clinic based rehabilitation. ${ }^{18,21-}$ ${ }^{24}$ After the 3-items are answered, the scores are tallied to determine a total score. ${ }^{18,21-24}$ A higher score is indicative of higher adherence. ${ }^{18,21-24}$

In a study conducted by Brewer ${ }^{21}$, SIRAS scores that were gathered by various practitioners of various education levels produced similar patient scores in a clinical setting. Strong interrater agreement was noted with rater agreement index (RAI) being .90 for high adherence, .86 for moderate, .84 for low, and aggregate $.87 .^{21}$ The limitation of a single participant in a hypothetical scenario, was a concern of this study, so another study was conducted. ${ }^{21}$ In this second study, Brewer utilized twelve patients who had anterior cruciate ligament reconstruction surgery. ${ }^{21}$ Two athletic trainers completed the SIRAS autonomously after four continuous rehabilitation sessions for each patient. ${ }^{21}$ The RAI value for the four appointments was .94 , indicating again a high level of interrater agreement for the SIRAS. ${ }^{21}$

It has been reported that the SIRAS has good internal consistency and test-retest reliability. ${ }^{21,24}$ The SIRAS has been deemed a strong psychometric measure in the literature. ${ }^{21,24}$ Compared to the RAdMAT, the SIRAS is a shorter tool that can be easily utilized after single or multiple sessions. ${ }^{18}$ However, the RAdMAT was slightly superior in addressing between group difference while evaluating adherence. ${ }^{18}$ The SIRAS can be utilized to find patients that are 
struggling to adhere to a rehabilitation program, thus indicating to the clinician to either change the rehabilitation program to fit the needs of the patient or implement psychological interventions. ${ }^{18,21-24}$

Another measure of rehabilitation adherence that has been discussed in the literature has been the Rehabilitation Adherence Questionnaire (RAQ). ${ }^{25}$ The RAQ is completed by the patient. ${ }^{25}$ It is a 40 -question survey that is completed utilizing a four point Likert scale ranging from strongly agree to strongly disagree. ${ }^{25}$ This questionnaire evaluates perceived exertion, pain tolerance, self-motivation, support from significant others, scheduling, and environmental conditions. ${ }^{18}$ This measurement tool has been utilized in retrospective clinic based research. ${ }^{25}$ A study completed reported high reported levels of each of the subscales resulted in higher adherence rates. ${ }^{25}$ In two other studies, higher levels of social support and pain tolerance lead to higher adherence rates. ${ }^{25}$ Although these studies exemplify consistency in results, there were limitations regarding the RAQ. ${ }^{25}$ The first limitation is that the RAQ has been utilized in retrospective studies and secondly there has been no reliability or validity testing on the RAQ. ${ }^{25}$ Therefore, it is uncertain if the subscales truly measure what is intended to measure. ${ }^{25}$

Brewer conducted a study to assess the psychometric properties of the RAQ. ${ }^{25}$ In his study patients who had undergone some type of knee surgery due to sport involvement were used. ${ }^{25}$ He utilized the 40-item RAQ, with patient attendance, the SIRAS, and a self-report of completion of home based exercise programs. ${ }^{25}$ The results of this study reported low levels for internal consistency coefficients at time one and two. ${ }^{25}$ The subscales of the RAQ also exemplified low Cronbach's alpha coefficients. ${ }^{25}$ In the second phase of the study, the RAQ was evaluated for content validity. ${ }^{25}$ The RAQ reported a lack of content validity. ${ }^{25}$ Ultimately the 
main findings of the study show that the RAQ is a weak psychometric assessment lacking internal consistency and criterion validity. ${ }^{25}$

The Rehabilitation Over Adherence Questionnaire (ROAQ) is a new assessment instrument that evaluates an athlete's likelihood to engage in activities and behaviors toward over adhering to the rehabilitation program. ${ }^{13}$ Over adherence has been defined as, "behaviors and underlying beliefs of athletes who engage in rehabilitation efforts that exceed practitionerrecommended guidelines."13 Examples of these behaviors include performing more exercises for prolonged periods of time that surpass the recommendations of the athletic trainer. ${ }^{13}$ This scale is composed of 10-items, which utilizes a 5-point Likert scale with 1 representing never and 5 representing always. ${ }^{13}$ There are two subscales in this measure, which include ignore practitioner recommendations and attempt an expedited rehabilitation. ${ }^{13}$

This is the only instrument reported in the literature that evaluated components regarding over adherence to the rehabilitation program. ${ }^{13}$ The measure was created utilizing the authors clinical experience, knowledge on the content, and research conducted on the topic. ${ }^{13}$ Four currently practicing certified athletic trainers were sent the questionnaire to evaluate the measures configuration, subject matter, and comprehensibility. ${ }^{13}$ Edits from the experts were added to the ROAQ for the first attempt at content validity. ${ }^{13}$ The experts believed that the 19 items measured the components of over adherence experienced in clinical practice and in the research. ${ }^{13}$ Construct validity was assessed using principal axis factoring (PAF) and confirmatory factor analysis (CFA). ${ }^{13}$

A majority of the adherence rehabilitation studies and literature have addressed under adherence in home-based exercises and the clinical setting. ${ }^{13}$ Limited studies have addressed over adherence to the rehabilitation program. ${ }^{13}$ However, it is not clearly stated in the literature 
what is considered ideal rehabilitation adherence. Similar to under adherence, over adherence can also cause prolonged return to play due to re-injury of the structure by doing too much too soon. ${ }^{13}$ By having a positive relationship with the athletic trainer, open communication, education of the healing and rehabilitation process, and recommendations can help to keep the athlete at an optimal level of adherence. ${ }^{13}$

Finally, the rehabilitation adherence measure for athletic training (RAdMAT) has been developed. ${ }^{18}$ The purpose of the RAdMAT is to measure adherence to the rehabilitation program in the athletic training setting. ${ }^{18}$ This is the only scale that is specific to the athletic training room. ${ }^{16}$ Due to nature of this scale it is only used with sport injuries. ${ }^{18}$

This measure was developed in three major steps including item generation, expert review, and an athletic trainer survey. ${ }^{18}$ The first step, item generation, involved seven currently practicing athletic trainers completing an online survey regarding adherence behaviors to rehabilitation. ${ }^{18}$ From the athletic trainers responses, two coders reviewed answers and developed a list of adherence behaviors. ${ }^{18}$ This list was sent back to the participants to check for accuracy. ${ }^{18}$

The next step in developing the RAdMAT was conducting an expert review. ${ }^{18}$ The adherence behaviors that were identified in step one were compiled into a survey format. ${ }^{18}$ The survey was then sent to twelve experts in the fields of athletic training education, sport psychologist, and rehabilitation research. ${ }^{18}$ The experts rated the content of the survey and made amendments in order to create effective questions addressing adherence. ${ }^{18}$ Researchers edited the original adherence survey to a 25-item RAdMAT. ${ }^{18}$

The final step of creating the RAdMAT was contacting athletic trainers to utilize the 25item RAdMAT measurement. ${ }^{18}$ Researchers instructed athletic trainers to complete the 25 -item 
RAdMAT for an athlete who was most adherent, least adherent, and average. ${ }^{18}$ The RAdMAT utilized a 4-point Likert scale with 1 representing never and 4 representing always. ${ }^{18}$ Scores ranged from 25 to 100 with higher scores representing greater adherence to rehabilitation programs. ${ }^{18}$ In conjunction with completing the RAdMAT, participants were also asked to complete the 3-item SIRAS. Scores for the SIRAS range from 3 to 15 with higher scores resulting in greater adherence. ${ }^{18}$ After statistical analysis; nine items were dropped for not providing additional information. ${ }^{18}$ The final RAdMAT consisted of 16 -items. ${ }^{18}$

The 16-item RAdMAT has three major subscales including attendance/participation, communication, and attitude/effort. ${ }^{18}$ Of those 16 questions, five evaluate attendance/participation; three assess communication, and eight evaluate attitude/effort components. ${ }^{18}$ The correlation analysis that was conducted for the RAdMAT and SIRAS resulted in a strong, positive, and significant relationship between the two measures. ${ }^{18}$ The RAdMAT is a psychologically sound measure and is thought to be more advantageous over the SIRAS in the athletic training room. ${ }^{18}$ The main advantage is the ability to distinguish differences of adherence levels between groups. ${ }^{18}$ The SIRAS is advantageous in the fact that it is a short measure, which does not require much time to complete. ${ }^{18,21}$ The RAdMAT is best utilized by certified athletic trainers who are currently practicing, however, results may not be able to be generalized due to a small sample size. ${ }^{18}$ However, there was strong support for internal consistency. ${ }^{18}$

The strengths in utilizing the RAdMAT is that it is specific to certified athletic trainers who are currently working in the collegiate setting. ${ }^{18}$ However, limitations of the RAdMAT include a small sample size, which affects generalizability. ${ }^{18}$ Another limitation includes low response rate, and utilizing recall cases for least, average, and most adherent. ${ }^{18}$ 
Finally, the Athletic Identity Measurement Scale (AIMS) is an assessment tool that evaluates the degree in which athletes classify themselves to their athletic portrayal. ${ }^{13,30,33,34}$ It utilizes a 7-point Likert scale ranging from 1 strongly disagree to 7 strongly agree. ${ }^{13,30,33,34}$ Originally, the AIMS was a 10-item assessment originally created by Brewer. ${ }^{30,33}$ However, even though it was widely accepted by researchers there were concerns regarding the measurement being one-dimensional or multidimensional. ${ }^{33}$ Brewer and Cornelius in 2001 tested the factorial structure and invariance of the 10-item AIMS. ${ }^{33}$ Three of the ten items were not sound measures and were removed from the 10-item AIMS, thus making it a 7-item assessment. ${ }^{33}$ The 7-item AIMS was then considered to be multidimensional with three main concepts being social identity, exclusivity, and negative affectivity. ${ }^{30,33,34}$

The social identity section, items one through three, evaluates how much the athlete views themselves as fulfilling the role of an athlete. ${ }^{34}$ The section of exclusivity, items four through six and nine, evaluates the self worth an individual experiences through participation in sport. ${ }^{34}$ And finally, negative affectivity, items eight and ten, assess the amount of negative thoughts and emotions experienced by an athlete when there is poor performance outcomes. ${ }^{34}$

This test has reported validity and reliability within an adolescent athletic population. ${ }^{13}$ In addition to high test-retest (0.89), internal consistency (0.80-0.93), concurrent validity, and construct valididty. ${ }^{13}$

In a study conducted by Visek et al. ${ }^{33}$, the goal was to further study the psychometric properties of the 7-item AIMS. AIMS was distributed to male collision sport Division I athletes. ${ }^{33}$ The results of their study supported the multidimensional structure of the 7-item AIMS, and that the assessment is valid and reliable while addressing athletic identity. ${ }^{33}$ 
A study conducted by Mills and Christensen ${ }^{34}$ evaluated athletic identity across sports and participation levels. Athletic identity scores were evaluated across elite, recreational, and non-athletes. ${ }^{34}$ Nineteen different sports were represented..$^{34}$ Using the 10 -item AIMS, scores ranged from 10 through 70, with higher scores representing higher levels of athletic identity and lower scores representing lower identification with athletic identity. ${ }^{34}$ The results indicated a gender difference in AIMS scores. ${ }^{34}$ Both male and female elite athletes reported higher AIMS scores compared to recreational and non-athletes. ${ }^{34}$ However, recreational athletes displayed higher scores compared to non-athletes. ${ }^{34}$

Athletic Trainers Perceptions of Adherence to Rehabilitation

Athletic trainers and physiotherapists have evaluated attitudes towards injured patients and athletes in regard to rehabilitation adherence. ${ }^{19,20,27,47}$ Health care professionals agree that the effectiveness of the rehabilitation programs affect return to play outcomes, and that it is a duty to help promote adherence. ${ }^{19,20,27,47}$ With over 200 variables that affect adherence rates, it is critical to uncover the most prominent variables that affect adherence. ${ }^{19,47}$ In addition, Granquist et al..$^{20}$ reported that $98.3 \%$ of athletic trainers reported poor rehabilitation adherence to be an issue in clinical practice. Further, $98.96 \%$ of athletic trainers reported working with athletes who poorly adhered to rehabilitation, and $97.9 \%$ reported that over adherence was an issue that was occasionally experienced in clinical practice..$^{20}$

A study conducted by Fisher et al. ${ }^{19}$ surveyed currently practicing athletic trainers. The responses can be categorized as injured athletic characteristics, environment, and athletic trainerathlete rapport. ${ }^{19,47}$ All of the respondents agreed that the relationship between the athletic trainer and the injured athlete is a vital component to the athlete adhering to the rehabilitation program in addition to injury and rehabilitation program education. ${ }^{19,47}$ Athletic trainers work 
with athletes on a daily basis and are the athlete's main source for information regarding the injury. ${ }^{19}$ Communication needs to be clear and at a level that the athlete understands. ${ }^{19}$

Athletic trainers have also reported that having the athletic training room accessible to injured athletes is important in promoting adherence. ${ }^{19}$ While planning rehabilitation sessions, it is important to take into account the injured athletes schedule in order to promote attendance. ${ }^{19}$

The final major theme was athlete's personality. ${ }^{19}$ Items such as pain tolerance, self motivation, and goals can help promote or be detrimental to the rehabilitation process. ${ }^{19} \mathrm{~A}$ certain degree of pain and discomfort is to be expected during rehabilitation sessions, however, some athletes do not understand what type of pain should be experienced during a rehabilitation session. ${ }^{19}$ Therefore, education on various types of pain is important to promote optimal adherence. $^{19}$

A study conducted by Byerly et al. ${ }^{47}$ evaluated Division II athletes who sustained a musculoskeletal injury that resulted in missing at least two days of training or competition. ${ }^{47}$ They reported 27 adherent athletes and 17 non-adherent athletes. ${ }^{47}$ The results were similar to Fisher et al. in that pain tolerance, social support, scheduling, self motivation, and environmental conditions all contributed to adherence rates. ${ }^{47}$

In the study that Granquist et al. ${ }^{20}$ conducted, the concept of the influence of injury and individual characteristics was elaborated. Injury specific factors, such as severity and rehabilitation length can affect adherence. ${ }^{20}$ Injuries with longer projected rehabilitations experienced decreased adherence levels. ${ }^{20}$ This could be due to the fact that making rehabilitation a part of the daily routine is difficult. ${ }^{23}$ In addition, factors such as level of competition, scholarship status, playtime, sport, and gender have shown differences in adherence levels. ${ }^{20}$ It was noted that older athletes were more compliant then younger counterparts. ${ }^{20}$ 
Females exemplified greater adherence levels compared to $\operatorname{men}^{20}$ Starters were more adherent due to being key contributors to team success compared to nonstarters. ${ }^{20}$ Individual sports and football were noted to have decreased adherence levels. ${ }^{20}$ The study also noted that adherence at the DIII level was a challenge, and that future research should be conducted to investigate adherence levels at lower collegiate levels. ${ }^{20}$

Playing through pain and injury have become highly prevalent and accepted in men's sports. ${ }^{28,30,31,45}$ This may be due to societal norms placed on men, such as playing through pain is normal. ${ }^{28}$ Men desire to appear masculine, however, when sports injury occurs it could be perceived that masculinity is decreased due to body deconditioning. ${ }^{28}$ Injury can make men feel weak, and this feeling of weakness could promote non-adherence to the rehabilitation program. ${ }^{28}$ This finding could help elaborate Granquist finding of women being more adherent to the rehabilitation program. ${ }^{20,28}$ However, Weinberg noted that through his study, women athletes were just as likely to adopt similar characteristics of males attempting to minimize pain and play though injury. ${ }^{28}$ Although women may be more likely to "buy into" the rehabilitation process than men. ${ }^{20}$

Pain tolerance has been reported as a factor in promoting optimal adherence. ${ }^{19,20,27,47}$ In a study conducted by Raudenbush et al. ${ }^{31}$, he reported that athletes report higher pain tolerance compared to non-athletes. He studied pain threshold in Division II athletes participating in contact and noncontact sports. ${ }^{31}$ He revealed that contact sports reported a higher pain tolerance compared to noncontact sports. ${ }^{31}$ Specifically men's lacrosse players had the lowest pain tolerance scores. ${ }^{31}$ Men's lacrosse and soccer players tolerated the pain for longer periods of times compared to the other teams. ${ }^{31}$ Athlete's who are competitive may be more adherent to 
rehabilitation regimens. ${ }^{31}$ Men's lacrosse players were the most competitive out of the sports teams with swimming being the least competitive. ${ }^{31}$

In conclusion, due to the reports of the epidemiology studies athletes competing in NCAA sports are constantly experiencing musculoskeletal injuries to the lower and upper extremity. ${ }^{35-44}$ Therefore, there is a need for higher adherence levels in order to have an optimal return to play. However, adherence levels have a broad range in various settings with a lack of a clear definition of optimal adherence levels. ${ }^{26,27}$ Theoretical frameworks have been developed in order to help guide and enhance adherence research. ${ }^{5-16}$ There are many different measurement tools to assess adherence, however, there is no gold standard stated in the literature. ${ }^{18,21-}$ 23,25,30,33,34 Utilizing a variety of measurement tools may help address issues regarding adherence, which could lead to the implementation of an intervention to promote more optimal adherence levels. ${ }^{18,21-23,25,30,33,34}$ Athletic trainers and physiotherapists have perceptions regarding positive and negative adherence to the rehabilitation program. ${ }^{19,20,47}$ Therefore, it is important as health care professionals to promoted adherence to the rehabilitation program in order to promote safe return to play protocols.

Summary

The literature shows that injuries occur across all sports in sanctioned NCAA competition. ${ }^{35-44}$ Further evaluation of injury rates, reported on time loss and non-time loss injuries. ${ }^{1}$ Theoretical frameworks help enhance to understanding of adherence by allowing research questions to be formed, hypothesis to be tested, and variables to be studied. ${ }^{5-14,16}$ There are many frameworks that are discussed in the literature such as attribution theory ${ }^{5}$, theory of planned behavior (TPB), ${ }^{5,7,16}$ health action process approach (HAPA), ${ }^{5,7,15}$ cognitive appraisal models, ${ }^{5,12-14}$ protection motivation theory $(\mathrm{PMT})^{5-10}$, and personal investment theory $(\mathrm{PIT})^{5,6,11}$. 
There is no gold standard for measuring adherence. The literature describes various different adherence measures that try to objectively assess injured patients adherence levels, which include the Sports Injury Rehabilitation Assessment Scale (SIRAS), ${ }^{18,21-23}$ Rehabilitation Adherence Questionnaire (RAQ) ${ }^{25}$ Rehabilitation Over Adherence Questionnaire (ROAQ) ${ }^{13}$ Rehabilitation Adherence Measure for Athletic Training (RAdMAT), ${ }^{18}$ and the Athletic Identity Measurement Scale (AIMS). ${ }^{13,30,33,34}$ Finally, athletic trainers and physiotherapist have various ideologies regarding their perceptions to injured athletes adherence to rehabilitation programs. ${ }^{19,20,27,47}$ However, the major emerging themes include athletic trainer influence, environmental conditions, athlete's personality, pain tolerance, self-motivation, and goals. ${ }^{19,20,27,47}$ 


\section{APPENDIX C}

\section{ADDITIONAL METHODS}

Table C1. Cover Letter to Athlete

December 3, 2015

Dear Participant,

This letter is a request for you to participate in a thesis project to assess adherence levels to the rehabilitation process. This research is being carried out by Kjersti Traaen; a graduate athletic training student at West Virginia University as well as the ATC/L at Margaret Belle Miller Middle School in Waynesburg, Pennsylvania. This research will be conducted under the supervision of Dr. Michelle Sandrey, the program director of the athletic training graduate program at West Virginia University. This research will fulfill the requirements for a Master's thesis project to complete a Master's degree in athletic training. Your participation in this project is greatly appreciated and will take approximately 10 to 15 minutes to fill out the attached questionnaires. These questionnaires will gather demographic information, athletic identity, and information regarding adherence to sport injury rehabilitation.

Your involvement in this research will be confidential, meaning that no indentifying information will be utilized. You must be 18 years of age or older to participate. Your participation in this research is completely voluntary and can withdraw at any time. You may skip any questions that do not wish to answer. Your athletic participation will not be affected if you decide either not to participate or to withdraw. West Virginia University's Institutional Review Board and Waynesburg University's Institutional Review Board have approved this study.

I hope that you will participate in this thesis project, as it could be beneficial in understanding the impact of adherence to the rehabilitation process in order to optimize return to play to sport.

Thank you very much for your time. Should you have any questions about this letter or the thesis project, please feel free to contact Kjersti Traaen at (717) 557-4074 or by e-mail at katraaen@mix.wvu.edu.

Thank you for your time and help with this project.

Sincerely, Kjersti Traaen ATC/L 
1. Please specify your gender
a. Male
b. Female

2. Please select the response that best corresponds to your age
a. 17 or younger
b. $18-22$
c. $23-27$
d. Over 27

3. Please specify what sports you participate
a. Football
b. Wrestling
c. Soccer
d. Baseball
e. Softball
f. Basketball
g. Lacrosse
h. Volleyball
i. Track and Field- indoor
j. Track and Filed- outdoor
k. Cross Country
1. Golf
m. Tennis

4. Please list in what sport did you sustain your injury:

5. How long have you participated in this sport
a. $0-5$ years
b. 6-10 years
c. 10-15 years
d. 15 or more years

6. Do you have a prior history of injury
a. Yes
b. No

7. Please specify your class status
a. Freshmen
b. Sophomore
c. Junior
d. Senior
e. Graduate

8. Please specify during what season did you sustain your injury
a. Preseason
b. In season
c. Post season 
1. To what extent do you ignore your athletic trainer's advice to avoid pushing through unwanted pain?

Never 12345 Always

2. To what extent do you ignore your athletic trainer's recommendations to avoid specific exercises or activities?

Never 12345 Always

3. To what extent do you avoid reporting pain to your athletic trainer?

Never 12345 Always

4. To what extent do you hide paid about your injury from doctors or other rehabilitation experts?

Never 12345 Always

5. To what extent do you ignore your athletic trainer's recommendations to avoid "doing too much too soon" in your rehabilitation?

Never 12345 Always

6. To what extent do you think that my family or teammates are concerned that I ignore my athletic trainer's advice to limit the rehabilitation exercises I perform?

Never 12345 Always

7. To what extent do you try to catch up with other athletes who are further ahead in their rehabilitation?

Never 12345 Always

8. To what extent do you think it is usually better to do too much rehabilitation than not enough? Never 12345 Always

9. To what extent do you perform more rehabilitation exercises than your athletic trainer recommends?

Never 12345 Always

10. To what extent do you believe I must progress quickly as possible in order to avoid losing physical fitness?

Never 12345 Always 


\section{C4. Athletic Identity Measurement Scale (AIMS)}

1. I consider myself an athlete strongly disagree 1234567 strongly agree

2. I have many goals related to sport strongly disagree 1234567 strongly agree

3. Most of my friends are athletes strongly disagree 1234567 strongly agree

4. Sport is the most important part of my life strongly disagree 1234567 strongly agree

5. I spend more time thinking about sport than anything else strongly disagree 1234567 strongly agree

6. I feel bad about myself when I do poorly in sport strongly disagree 1234567 strongly agree

7. I would be very depressed if I were injured and could not compete in sport strongly disagree 1234567 strongly agree 
December 3, 2015

Dear Participant,

You have been selected to participate in this research study based on your credentials and experience with sport injury rehabilitation. Working as a certified athletic trainer in the Division III setting in Waynesburg Pennsylvania qualifies you for this study. My name is Kjersti Traaen, and I am a graduate student in the West Virginia University Athletic Training Program along with my GA assignment at Margret Miller Middle School. I will be conducting a study with the primary investigator, Michelle A. Sandrey, PhD, ATC to fulfill requirements for a Master's thesis project and to complete a Masters of Science degree in Athletic Training. All are affiliated with West Virginia University.

This research will require you to complete the 3-item Sport Injury Rehabilitation Adherence Scale (SIRAS) and the Rehabilitation Adherence Measure for Athletic Training (RAdMAT) on a bi-weekly basis assessing the athletes' intensity, frequency, and receptiveness to instruction regarding the rehabilitation program prescribed by the athletic trainer. This measure utilizes a Likert scale and will take approximately ten minutes to complete. A hard copy will be provided to you.

This is a completely voluntary activity and all responses are guaranteed to be anonymous and confidential. Questions can be skilled, and you have the right to withdraw any data you submit at any time. Your job status will not be affected by failure to participate. West Virginia University and Waynesburg University IRB has approved and the approval is on file. If you have any questions or concerns please contact me at katraaen@mix.wvu.edu. You can contact Michelle A Sandrey, PhD, ATC, Principal Investigator and Graduate Athletic Training Program Director at West Virginia University at msandrey@ mail.wvu.edu.

Thank you for your time and help with this project.

Sincerely,

Kjersti Traaen ATC/L 


\section{C6. Sport Injury Rehabilitation Adherence Scale (SIRAS)}

1. Circle the number that best indicates the intensity with which this patient completed rehabilitation exercises during today's appointment:

minimum effort 12345 maximum effort

2. During today's appointment, how frequently did this patient follow your instructions and advice?

never 12345 always

3. How receptive was this patient to changes in the rehabilitation program during today's appointment?

very unreceptive 12345 very receptive 
Please rate the athlete on each item using the scale: $1=$ never, $2=$ occasionally, 3

$=$ often, 4 = always

1. Attends scheduled rehabilitation sessions

1234

2. Arrives at rehabilitation on time

1234

3. Follows the athletic trainer's instructions during rehabilitation sessions

1234

4. Follows the prescribed rehabilitation plan

1234

5. Completes all tasks assigned by the athletic trainer

1234

6. Asks questions about his or her rehabilitation

1234

7. Communicates with the athletic trainer if there is a problem with the exercises

1234

8. Provides the athletic trainer feedback about the rehabilitation program

1234

9. Has a positive attitude during rehabilitation sessions

1234

10. Has a positive attitude toward the rehabilitation process

1234

11. Gives $100 \%$ effort in rehabilitation sessions

1234

12. Is self-motivated in rehabilitation sessions

1234

13. Is an active participant in the rehabilitation process

1234

14. Stays focused while doing rehabilitation exercises

1234

15. Is motivated to complete rehabilitation

1234

16. Shows interest in the rehabilitation process

1234 


\section{APPENDIX D}

\section{ADDITIONAL RESULTS}

Table D1. Demographic Information

\begin{tabular}{|c|c|c|}
\hline Characteristic & Percent & \\
\hline \multicolumn{3}{|l|}{$\begin{array}{l}\text { Characteristic } \\
\text { Age }\end{array}$} \\
\hline 17 or younger & $0 \%$ & 0 \\
\hline $18-22$ & $100 \%$ & 0 \\
\hline $23-26$ & $0 \%$ & 0 \\
\hline 27 or older & $0 \%$ & 0 \\
\hline \multicolumn{3}{|l|}{ Gender } \\
\hline Female & $8.3 \%$ & 1 \\
\hline Male & $91.7 \%$ & 11 \\
\hline \multicolumn{3}{|l|}{ Sport Participation } \\
\hline Football & $58.3 \%$ & 7 \\
\hline Soccer & $16.7 \%$ & 2 \\
\hline Baseball & $8.3 \%$ & 1 \\
\hline Multiple Sports & $16.7 \%$ & 2 \\
\hline \multicolumn{3}{|l|}{ Sport When Injury } \\
\hline \multicolumn{3}{|l|}{ Occurred } \\
\hline Football & $58.3 \%$ & 7 \\
\hline Soccer & $16.7 \%$ & 2 \\
\hline Indoor Track & $8.3 \%$ & 1 \\
\hline Baseball & $8.3 \%$ & 1 \\
\hline Intramurals & $8.3 \%$ & 1 \\
\hline \multicolumn{3}{|l|}{ Years of Participation } \\
\hline $0-5$ years & $16.7 \%$ & 2 \\
\hline 6-10 & $8.3 \%$ & 1 \\
\hline 10-15 & $41.7 \%$ & 5 \\
\hline 15 or more & $33.3 \%$ & 4 \\
\hline \multicolumn{3}{|l|}{ History of Injury } \\
\hline Yes & $50 \%$ & 6 \\
\hline No & $50 \%$ & 6 \\
\hline \multicolumn{3}{|l|}{ Class Status } \\
\hline Freshmen & $41.7 \%$ & 5 \\
\hline Sophomore & $33.3 \%$ & 4 \\
\hline Junior & $25 \%$ & 3 \\
\hline Senior & $0 \%$ & 0 \\
\hline \multicolumn{3}{|c|}{ Season Injury Occurred } \\
\hline Pre-Season & $0 \%$ & 0 \\
\hline In-Season & $83.3 \%$ & 10 \\
\hline Post-Season & $16.7 \%$ & 2 \\
\hline
\end{tabular}


Table D2. Repeated Measures ANOVA 1x2 ROAQ, SIRAS, and RAdMAT

\begin{tabular}{lll} 
Measure & F-Value & Significance \\
\hline ROAQ & 2.382 & .151 \\
SIRAS & .786 & .394 \\
RAdMAT & .592 & .458
\end{tabular}

KEY: *significant findings at $\mathrm{P}<.05$

Table D3. Correlation Analysis for AIMS \& ROAQ

\begin{tabular}{lll} 
Measure & $\begin{array}{l}\text { ROAQ Pre-test Pearson } \\
\text { Correlation }\end{array}$ & $\begin{array}{l}\text { ROAQ Post-test Pearson } \\
\text { Correlation }\end{array}$ \\
\hline AIMS \& ROAQ & .314 & .319
\end{tabular}

Table D4. Correlation Analysis for AIMS \& Years of Participation

$\begin{array}{lll}\text { Measure } & \text { Pearson Correlation } & \text { Significance }\end{array}$

\begin{tabular}{lll}
\hline $\begin{array}{l}\text { AIMS \& Years of } \\
\text { Participation }\end{array}$ & .353 & .261 \\
& \\
\hline
\end{tabular}

Table D5. Correlation Analysis for SIRAS \& RAdMAT

\begin{tabular}{lll}
\hline Measure & Pearson Correlation & Significance \\
\hline Pre SIRAS \& Pre & .932 & $\mathrm{p}<0.001$ \\
RAdMAT & & 0.004 \\
$\begin{array}{l}\text { Post SIRAS \& Pre } \\
\text { RAdMAT }\end{array}$ & .764 & 0.009 \\
$\begin{array}{l}\text { Pre SIRAS \& Post } \\
\text { RAdMAT }\end{array}$ & .714 & 0.016 \\
$\begin{array}{l}\text { Post SIRAS \& Post } \\
\text { RAdMAT }\end{array}$ & .673 & $\mathrm{p}<0.001$ \\
$\begin{array}{l}\text { Total SIRAS \& Total } \\
\text { RAdMAT }\end{array}$ & 0.901 & \\
\hline
\end{tabular}


Figure D1. Sport Participation

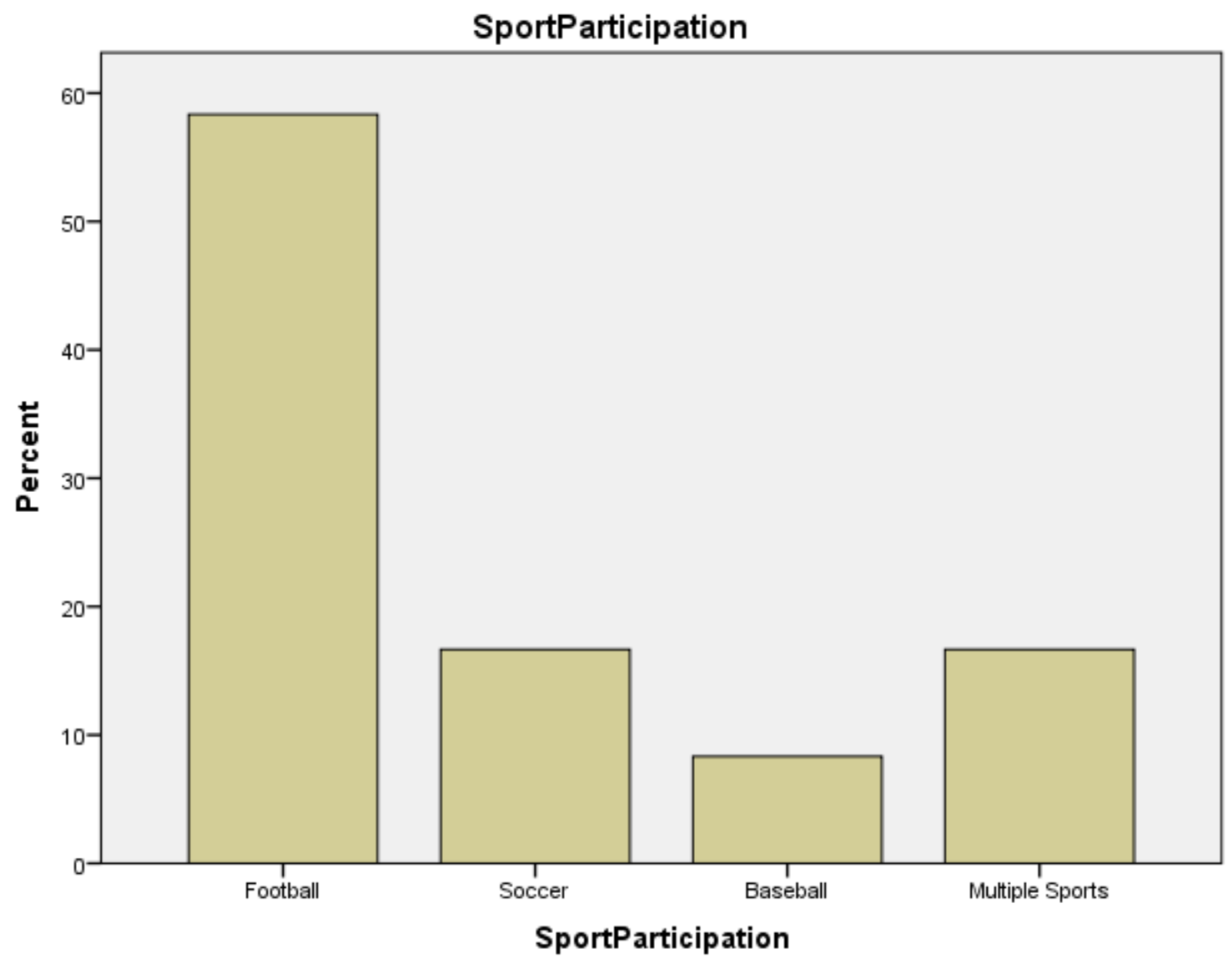

Figure D2. Sport Injury was Sustained

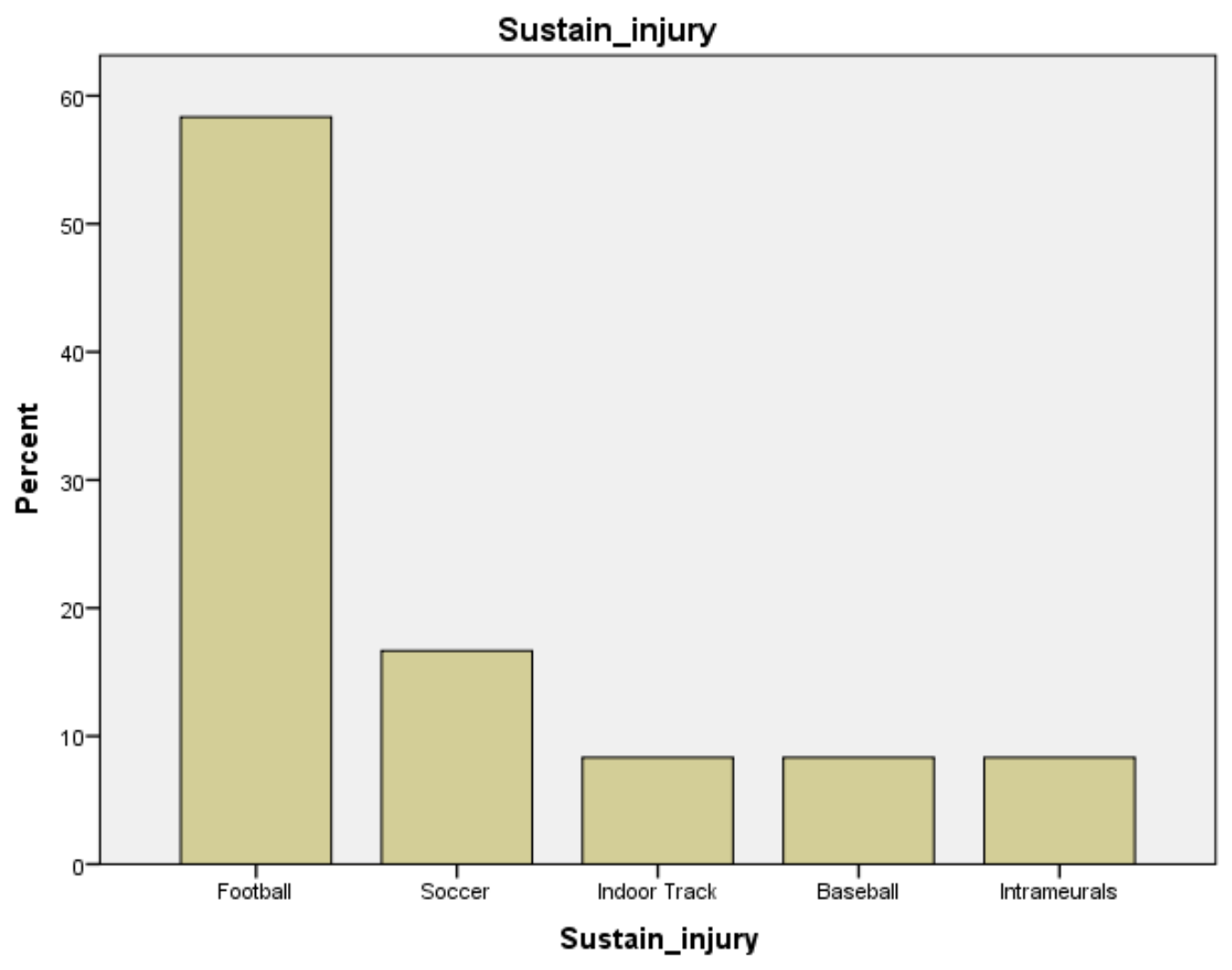


Figure 3D. Years of Sport Participation

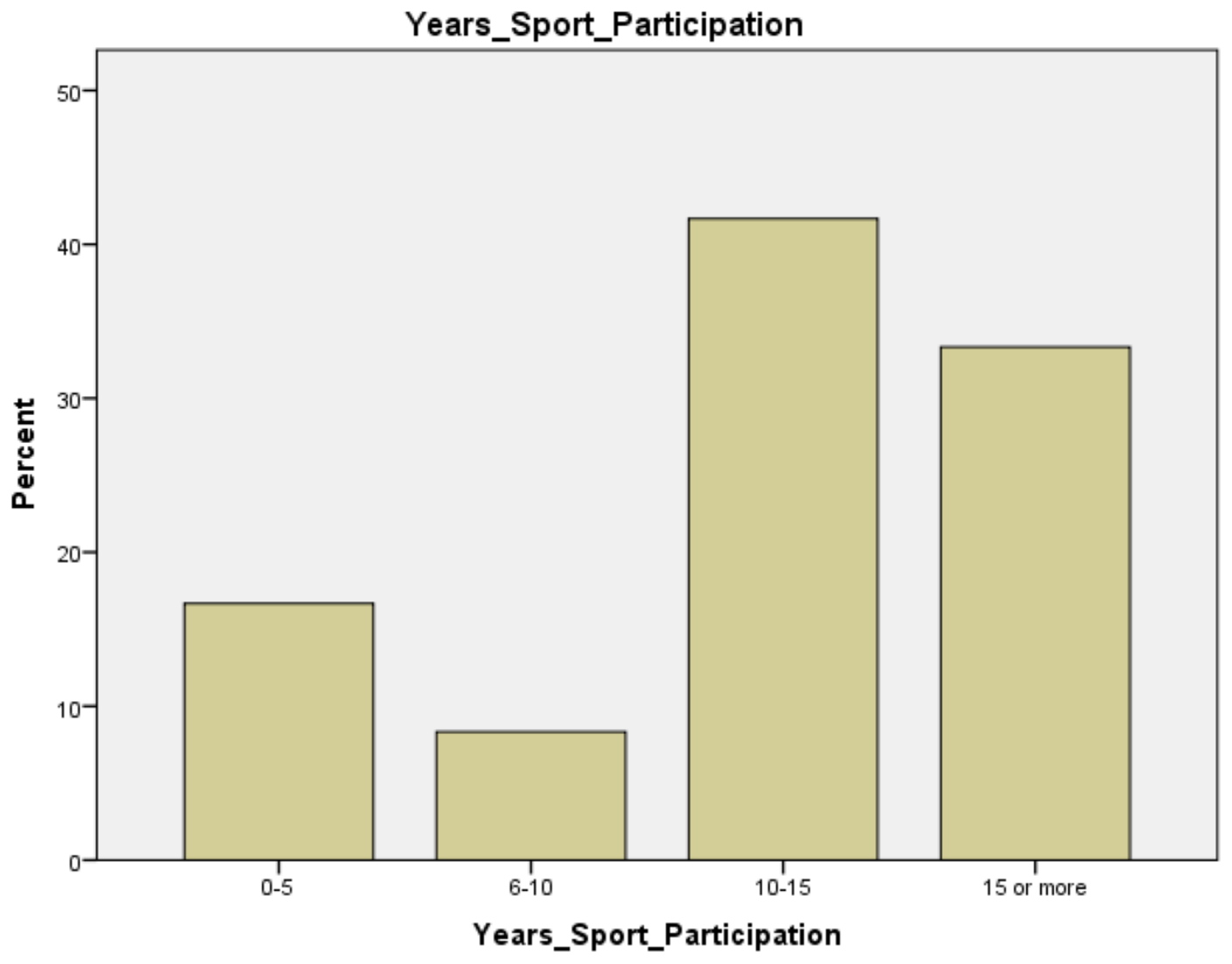




\section{APPENDIX E}

\section{RECOMMENDATIONS FOR FUTURE RESEARCH}

1. A recommendation would be to repeat this study involving more Division III institutions within the same competitive conference

2. Another recommendation would be to repeat this study over the course of an athletic season in order to increase diversity in the sample size and to follow extensive time-loss injuries that may have required surgery.

3. In order to have more subjects, IRB approval should have been completed sooner.

4. In addition, the definition of an injury should have been changed in order for more participants to qualify to participate in the study. In order to include more time-loss injuries, the definition should be decreased days of restricted participation. 


\section{ADDITIONAL REFERENCES}

35. Dick R, Putukian M, Agel J, Evans T, Marshall S. Descriptive epidemiology of collegiate women's soccer injuries: National collegiate athletic association injury surveillance system, 1988-1989 through 2002-2003. J Athl Train. 2007;42(2):278-285.

36. Agel J, Evans T, Dick R, Putukian M, Marshall S. Descriptive epidemiology of collegiate men's soccer injuries: National collegiate athletic association injury surveillance system, 1988-1989 through 2002-2003. J Athl Train. 2007;42(2):270-277.

37. Agel J, Olson D, Dick R, Arendt E, Marshall S, Sikka R. Descriptive epidemiology of collegiate women's basketball injuries: National collegiate athletic association injury surveillance system, 1988-1989 through 2003-2004. J Athl Train. 2007;42(2):202-210.

38. Dick R, Hertel J, Agel J, Grossman J, Marshall S. Descriptive epidemiology of collegiate men's basketball injuries: national collegiate athletic association injury surveillance system, 1988-1989 through 2003-2004. J Athl Train. 2007;42(2):194-201.

39. Marshall S, Hamstra-Wright K, Dick R, Grove K, Agel J. Descriptive epidemiology of collegiate women's softball injuries: National collegiate athletic association injury surveillance system, 1988-1989 through 2003-2004. J Athl Train. 2007;42(2):286-294.

40. Dick R, Sauers E, Agel J, Keuter G, Marshall S, McCarty K. Descriptive epidemiology of collegiate men's baseball injuries: national collegiate athletic association injury surveillance system, 1988-1989 through 2003-2004. J Athl Train. 2007;42(2):183-193.

41. Dick R, Lincoln A, Agel J, Carter E, Marshall S, Hinton R. Descriptive epidemiology of collegiate women's lacrosse injuries: National collegiate athletic association injury surveillance system, 1988-1989 through 2003-2004. J Athl Train. 2007; 42(2): 262-269.

42. Agel J, Palmieri-Smith R, Dick R, Wojtys E, Marshall S. Descriptive epidemiology of collegiate women's volleyball injuries: National collegiate athletic association injury surveillance system, 1988-1989 through 2003-2004. J Athl Train. 2007;42(2):295-302.

43. Dick R, Ferrara M, Reifsteck F, Agel J, Courson R, Marshall S, Hanley M, Reifsteck F. Descriptive epidemiology of collegiate men's football injuries: National collegiate athletic association injury surveillance system, 1988-1989 through 2003-2004. J Athl Train. 2007;42(2):221-233.

44. Agel J, Ransone J, Dick R, Oppliger R, Marshall S. Descriptive epidemiology of collegiate men's wrestling injuries: national collegiate athletic association injury surveillance system, 1988-1989 through 2003-2004. J Athl Train. 2007;42(2):303-310.

45. Nixon, H. A social network analysis of influences on athletes to play with pain and injuries. J Sport Social Issues. 1992;16:127-135. 
46. Nixon H. Accepting the risks of pain and injury in sport: mediated cultural influences on playing hurt. Socio Sport J. 1993;10(2):183-196.

47. Byerly P, Worrell T, Cahimer J, Domholdt E. Rehabilitation compliance in an athletic training environment. J Athl Train. 1994: 29(4); 352-355. 